\title{
Higher order numerical methods for solving fractional differential equations
}

\author{
Yubin Yan · Kamal Pal · Neville J Ford
}

Received: date / Accepted: date

\begin{abstract}
In this paper we introduce higher order numerical methods for solving fractional differential equations. We use two approaches to this problem. The first approach is based on a direct discretisation of the fractional differential operator: we obtain a numerical method for solving a linear fractional differential equation with order $0<\alpha<1$. The order of convergence of the numerical method is $O\left(h^{3-\alpha}\right)$. Our second approach is based on discretisation of the integral form of the fractional differential equation and we obtain a fractional Adams-type method for a nonlinear fractional differential equation of any order $\alpha>0$. The order of convergence of the numerical method is $O\left(h^{3}\right)$ for $\alpha \geq 1$ and $O\left(h^{1+2 \alpha}\right)$ for $0<\alpha \leq 1$ for sufficiently smooth solutions. Numerical examples are given to show that the numerical results are consistent with the theoretical results.
\end{abstract}

Keywords Fractional differential equation - finite difference method - Caputo fractional derivative $\cdot$ error estimates

Mathematics Subject Classification (2000) 26A33 $\cdot$ 65L70 $\cdot$ 65L05

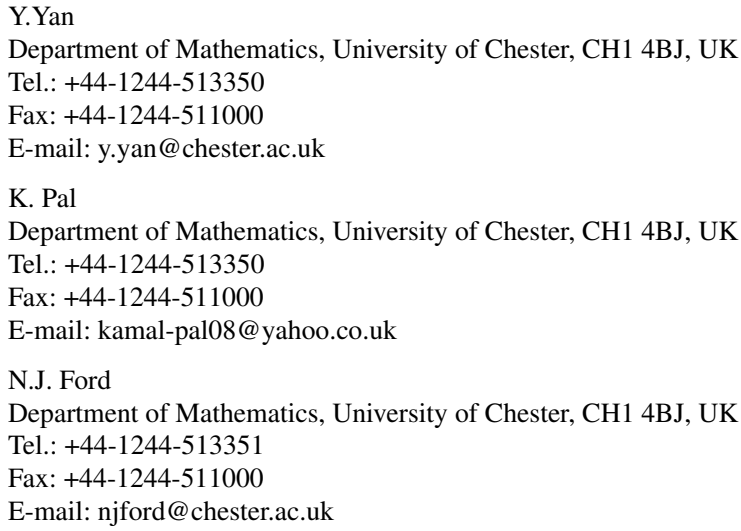




\section{Introduction}

We consider numerical methods for solving the fractional differential equation

$$
\begin{aligned}
& { }_{0}^{C} D_{t}^{\alpha} y(t)=f(t, y(t)), \quad 0<t<T, \\
& y^{(k)}(0)=y_{0}^{(k)}, \quad k=0,1,2, \ldots,\lceil\alpha\rceil-1,
\end{aligned}
$$

where the $y_{0}^{(k)}$ may be arbitrary real numbers and $\alpha>0$. Here ${ }_{0}^{C} D_{t}^{\alpha}$ denotes the differential operator in the sense of Caputo denoted by

$$
{ }_{0}^{C} D_{t}^{\alpha} y(t)=\frac{1}{\Gamma(n-\alpha)} \int_{0}^{t}(t-u)^{n-\alpha-1} y^{(n)}(u) d u,
$$

where $n=\lceil\alpha\rceil$ is the smallest integer $\geq \alpha$.

Existence and uniqueness of solutions for (1.1) -(1.2) have been studied, for example, in Podlubny [21], Diethelm [8], Diethelm and Ford [10]. Numerical methods for solving fractional differential equations have been considered by many authors and we mention here a few key contributions. Lubich [18] wrote the fractional differential equation in the form of an Abel-Volterra integral equation and used the convolution quadrature method to approximate the fractional integral and obtained approximate solutions of the fractional differential equation. Diethelm [7] wrote the fractional Riemann-Liouville derivative by using the Hadamard finite-part integral and approximated the integral by using a quadrature formula and obtained an implicit numerical algorithm for solving a linear fractional differential equation. Diethelm and Luchko [13] used the observation that a fractional differential equation has an exact solution, which can be expressed as a Mittag-Leffler type function. Then they used convolution quadrature and discretised operational calculus to produce an approximation to this Mittag-Leffler function. Blank [1] applied a collocation method to approximate the fractional differential equation. Podlubny [21] used the Grünwald and Letnikov method to approximate the fractional derivative and defined an implicit finite difference method for solving (1.1)-(1.2) and proved that the order of convergence is $O(h)$, where $h$ is the step size. Gorenflo [17] introduced a second order $O\left(h^{2}\right)$ difference method for solving (1.1)-(1.2), but the conditions to achieve the desired accuracy are restrictive. In [11], the authors approximated the integral in (1.5) by using a piecewise linear interpolation polynomial and introduced a fractional Adams-type predictor-corrector method for solving (1.1)(1.2), proving that the order of convergence of the numerical method is $\min \{2,1+\alpha\}$ for $0<\alpha \leq 2$ if ${ }_{0}^{C} D_{t}^{\alpha} y \in C^{2}[0, T]$. Deng [3] modified the method in [11] and introduced a new predictor-corrector method for solving (1.1)-(1.2) and the convergence order is proved to be $\min \{2,1+2 \alpha\}$ for $\alpha \in(0,1]$. In [22], the authors introduced a so-called Jacobi-predictor-corrector approach to solve (1.1)-(1.2) which is based on the polynomial interpolation and the Gauss-Lobatto quadrature with respect to some Jacobi-weight function and the computational cost is $O(N), N=1 / h$ and any desired convergence order can be obtained. In [2], a high order numerical method for solving (1.1)-(1.2) is obtained where a quadratic interpolation polynomial was used to approximate the integral. Ford, Morgado and Rebelo recently (see [16]) used a nonpolynomial collocation method to achieve good convergence properties without 
assuming any smoothness of the solution. There are also several works that are related to the fixed memory principle and the nested memory concept for solving (1.1)-(1.2), see, e.g., [15], [12], [3], [4], [5], etc.

Two approaches are used in this paper to develop new numerical methods for solving (1.1)- (1.2). The first approach is based on a direct discretisation of the fractional differential operator in the form proposed by Diethelm [6]. In [7], Diethelm considered the following linear fractional differential equation, with $0<\alpha<1$,

$$
\begin{aligned}
& { }_{0}^{C} D_{t}^{\alpha} y(t)=\beta y(t)+f(t), \quad 0 \leq t \leq 1, \\
& y(0)=y_{0},
\end{aligned}
$$

where $\beta<0, f$ is a given function on the interval $[0,1]$. Diethelm [7] used a firstdegree compound quadrature formula to approximate the Hadamard finite-part integral in the equivalent form of (1.3)-(1.4) and defined a numerical method for solving (1.3)-(1.4) and proved that the order of convergence of the numerical method is $O\left(h^{2-\alpha}\right), 0<\alpha<1$. Here we approximate the Hadamard finite-part integral by using the second-degree compound quadrature formula and obtain an asymptotic expansion of the error for solving (1.3)-(1.4), which implies that the order of convergence of the numerical method is $O\left(h^{3-\alpha}\right), 0<\alpha<1$. Moreover, a high order finite difference method $\left(O\left(h^{3-\alpha}\right), 0<\alpha<2\right)$ for approximating the Riemann-Liouville fractional derivative is given, which may be applied to construct high order numerical methods for solving time-space-fractional partial differential equations.

Our second approach for solving the fractional differential equation (1.1)-(1.2) is based on the discretisation of the integral in the equivalent form of (1.1)-(1.2), see [11]. It is well-known that (1.1)-(1.2) is equivalent to the Volterra integral equation

$$
y(t)=\sum_{v=0}^{\lceil\alpha\rceil-1} y_{0}^{(v)} \frac{t^{\nu}}{v !}+\frac{1}{\Gamma(\alpha)} \int_{0}^{t}(t-u)^{\alpha-1} f(u, y(u)) d u
$$

We use piecewise quadratic interpolation polynomials to approximate the integral in (1.5) and introduce a high order fractional Adams method for solving (1.5) and prove that the order of convergence of the numerical method is $\min \{3,1+2 \alpha\}$ for $\alpha \in(0,2]$ if ${ }_{0}^{C} D_{t}^{\alpha} y(t) \in C^{3}[0, T]$. This method has higher convergence order than the method in [3]. It is easier to implement our numerical algorithm compared with the method in [22] where the Jacobi-Gauss-Lobatto nodes must be calculated at each time level. Our method is simpler than the method in [2] in the sense that we are using a predictor-corrector method and therefore we do not need to solve the nonlinear system at each time level.

The paper is organised as follows: in Section 2, we consider how we can extend the Diethelm method for solving a linear fractional differential equation. In Section 3 , we consider the fractional Adams method for solving a nonlinear fractional differential equation. Finally in Section 4, we consider some numerical examples. 


\section{Extending Diethelm's method}

In this section we will consider a higher order numerical method for solving (1.3)(1.4). It is well-known that (1.3)-(1.4) is equivalent, with $0<\alpha<1$, to the following problem:

$$
{ }_{0}^{R} D_{t}^{\alpha}\left[y(t)-y_{0}\right]=\beta y(t)+f(t), \quad 0 \leq t \leq 1,
$$

where ${ }_{0}^{R} D_{t}^{\alpha} y(t)$ denotes the Riemann-Liouville fractional derivative defined by, with $0<\alpha<1$,

$$
{ }_{0}^{R} D_{t}^{\alpha} y(t)=\frac{1}{\Gamma(1-\alpha)} \frac{d}{d t} \int_{0}^{t}(t-u)^{-\alpha} y(u) d \tau .
$$

The Riemann-Liouville fractional derivative ${ }_{0}^{R} D_{t}^{\alpha} y(t)$ can be written as [7]

$$
{ }_{0}^{R} D_{t}^{\alpha} y(t)=\frac{1}{\Gamma(-\alpha)} \oint_{0}^{t}(t-u)^{-1-\alpha} y(u) d u
$$

where the integral $\oint$ denotes the Hadamard finite-part integral.

In [7], Diethelm approximated the Hadamard finite-part integral in (2.3) by piecewise linear interpolation polynomials and defined a numerical method for solving (2.1). In this section, we will approximate the Hadamard finite-part integral by using piecewise quadratic interpolation polynomials.

Let $M$ be a fixed positive integer and let $0=t_{0}<t_{1}<t_{2}<\cdots<t_{2 j}<t_{2 j+1}<\cdots<$ $t_{2 M}=1$ be a partition of $[0,1]$ and $h$ the step size. At node $t_{2 j}=\frac{2 j}{2 M}$, the equation (2.1) satisfies

$$
{ }_{0}^{R} D_{t}^{\alpha}\left[y\left(t_{2 j}\right)-y_{0}\right]=\beta y\left(t_{2 j}\right)+f\left(t_{2 j}\right), \quad j=1,2, \ldots, M,
$$

and at node $t_{2 j+1}=\frac{2 j+1}{2 M}$, the equation (2.1) satisfies

$$
{ }_{0}^{R} D_{t}^{\alpha}\left[y\left(t_{2 j+1}\right)-y_{0}\right]=\beta y\left(t_{2 j+1}\right)+f\left(t_{2 j+1}\right), \quad j=0,1,2, \ldots, M-1 .
$$

Let us first consider the discretisation of (2.4). Note that

${ }_{0}^{R} D_{t}^{\alpha} y\left(t_{2 j}\right)=\frac{1}{\Gamma(-\alpha)} \oint_{0}^{t_{2 j}}\left(t_{2 j}-\tau\right)^{-1-\alpha} y(\tau) d \tau=\frac{t_{2 j}^{-\alpha}}{\Gamma(-\alpha)} \oint_{0}^{1} w^{-1-\alpha} y\left(t_{2 j}-t_{2 j} w\right) d w$.

For every $j$, we replace $g(w)=y\left(t_{2 j}-t_{2 j} w\right)$ in the integral in (2.6) by a piecewise quadratic interpolation polynomial with equispaced nodes $0, \frac{1}{2 j}, \frac{2}{2 j}, \ldots, \frac{2 j}{2 j}$. We then have

$$
\oint_{0}^{1} w^{-1-\alpha} g(w) d w=\oint_{0}^{1} w^{-1-\alpha} g_{2}(w) d w+R_{2 j}(g),
$$

where $g_{2}(w)$ is the piecewise quadratic interpolation polynomial of $g(w)$ with equispaced nodes $0, \frac{1}{2 j}, \frac{2}{2 j}, \ldots, \frac{2 j}{2 j}$ and $R_{2 j}(g)$ is the remainder term. 
Lemma 2.1 Let $0<\alpha<1$. We have

$$
\oint_{0}^{1} w^{-1-\alpha} g_{2}(w) d w=\sum_{k=0}^{2 j} \alpha_{k, 2 j} g\left(\frac{k}{2 j}\right)
$$

where

$$
\begin{aligned}
& (-\alpha)(-\alpha+1)(-\alpha+2)(2 j)^{-\alpha} \alpha_{l, 2 j}=\left\{\begin{array}{lr}
2^{-\alpha}(\alpha+2), & \text { for } l=0, \\
(-\alpha) 2^{2-\alpha}, & \text { for } l=1, \\
(-\alpha)\left(-2^{-\alpha} \alpha\right)+\frac{1}{2} F_{0}(2), & \text { for } l=2, \\
-F_{1}(k), & \text { for } l=2 k-1, \\
& k=2,3, \ldots, j, \\
\frac{1}{2}\left(F_{2}(k)+F_{0}(k+1)\right), & \text { for } l=2 k, \\
& k=2,3, \ldots, j-1, \\
\frac{1}{2} F_{2}(j), & \text { for } l=2 j,
\end{array}\right. \\
& F_{0}(k)=(2 k-1)(2 k)\left((2 k)^{-\alpha}-(2 k-2)^{-\alpha}\right)(-\alpha+1)(-\alpha+2) \\
& -((2 k-1)+2 k)\left((2 k)^{-\alpha+1}-(2 k-2)^{-\alpha+1}\right)(-\alpha)(-\alpha+2) \\
& +\left((2 k)^{-\alpha+2}-(2 k-2)^{-\alpha+2}\right)(-\alpha)(-\alpha+1), \\
& F_{1}(k)=(2 k-2)(2 k)\left((2 k)^{-\alpha}-(2 k-2)^{-\alpha}\right)(-\alpha+1)(-\alpha+2) \\
& -((2 k-2)+2 k)\left((2 k)^{-\alpha+1}-(2 k-2)^{-\alpha+1}\right)(-\alpha)(-\alpha+2) \\
& +\left((2 k)^{-\alpha+2}-(2 k-2)^{-\alpha+2}\right)(-\alpha)(-\alpha+1),
\end{aligned}
$$

and

$$
\begin{aligned}
F_{2}(k)= & (2 k-2)(2 k-1)\left((2 k)^{-\alpha}-(2 k-2)^{-\alpha}\right)(-\alpha+1)(-\alpha+2) \\
& -((2 k-2)+(2 k-1))\left((2 k)^{-\alpha+1}-(2 k-2)^{-\alpha+1}\right)(-\alpha)(-\alpha+2) \\
& +\left((2 k)^{-\alpha+2}-(2 k-2)^{-\alpha+2}\right)(-\alpha)(-\alpha+1) .
\end{aligned}
$$

Proof For fixed $2 j$, let $0<\frac{1}{2 j}<\frac{2}{2 j}<\cdots<\frac{2 j}{2 j}=1$ be a partition of $[0,1]$. Denote $w_{l}=\frac{l}{2 j}, l=0,1,2, \ldots, 2 j$. We then have, for $k=1,2, \ldots, j$,

$$
\begin{aligned}
g_{2}(w)= & \frac{\left(w-w_{2 k-1}\right)\left(w-w_{2 k}\right)}{\left(w_{2 k-2}-w_{2 k-1}\right)\left(w_{2 k-2}-w_{2 k}\right)} g\left(w_{2 k-2}\right) \\
& +\frac{\left(w-w_{2 k-2}\right)\left(w-w_{2 k}\right)}{\left(w_{2 k-1}-w_{2 k-2}\right)\left(w_{2 k-1}-w_{2 k}\right)} g\left(w_{2 k-1}\right) \\
& +\frac{\left(w-w_{2 k-2}\right)\left(w-w_{2 k-1}\right)}{\left(w_{2 k}-w_{2 k-2}\right)\left(w_{2 k}-w_{2 k-1}\right)} g\left(w_{2 k}\right), \quad \text { for } w \in\left[w_{2 k-2}, w_{2 k}\right] .
\end{aligned}
$$


Let us now consider

$$
\oint_{0}^{1} w^{-1-\alpha} g_{2}(w) d w=\left[\oint_{0}^{w_{2}}+\int_{w_{2}}^{w_{4}}+\cdots+\int_{w_{2 j-2}}^{w_{2 j}}\right] w^{-1-\alpha} g_{2}(w) d w .
$$

By the definition of the Hadamard finite-part integral [6], we obtain

$$
\begin{aligned}
\oint_{0}^{w_{2}} w^{-1-\alpha} g_{2}(w) d w & =\frac{g_{2}(0)\left(w_{2}\right)^{-\alpha}}{-\alpha}+\int_{0}^{w_{2}} w^{-1-\alpha}\left[\int_{0}^{w} g_{2}^{\prime}(y) d y\right] d w \\
& =\frac{2^{-\alpha}}{(-\alpha)(2 j)^{-\alpha}} g_{2}(0)+\int_{0}^{w_{2}} w^{-1-\alpha}\left(g_{2}(w)-g_{2}(0)\right) d w .
\end{aligned}
$$

By using (2.9), we have

$$
\begin{aligned}
\oint_{0}^{w_{2}} g_{2}(w) w^{-1-\alpha} d w \\
=\frac{2^{-\alpha}}{(-\alpha)(2 j)^{-\alpha}} g(0)+\int_{0}^{w_{2}} w^{-1-\alpha}\left[\frac{(2 j)^{2}}{2}\left(w^{2}-\left(w_{1}+w_{2}\right) w\right) g(0)\right. \\
\left.\quad+\frac{(2 j)^{2}}{-1}\left(w^{2}-\left(0+w_{2}\right) w\right) g\left(w_{1}\right)+\frac{(2 j)^{2}}{2}\left(w^{2}-\left(0+w_{1}\right) w\right) g\left(w_{2}\right)\right] d w \\
=\frac{2^{-\alpha}(\alpha+2)}{(-\alpha)(-\alpha+1)(-\alpha+2)(2 j)^{-\alpha}} g(0)+\frac{2^{2-\alpha}}{(-\alpha+1)(-\alpha+2)(2 j)^{-\alpha}} g\left(w_{1}\right) \\
\quad+\frac{-2^{-\alpha} \alpha}{(-\alpha+1)(-\alpha+2)(2 j)^{-\alpha}} g\left(w_{2}\right) .
\end{aligned}
$$

Similarly we have, after a simple calculation,

$$
\begin{aligned}
(-\alpha)(-\alpha+1)(-\alpha+2)(2 j)^{-\alpha} \int_{w_{2 k}}^{w_{2 k+2}} g_{2}(w) w^{-1-\alpha} d w \\
=\frac{1}{2} F_{0}(k) g\left(w_{2 k-2}\right)+(-1) F_{1}(k) g\left(w_{2 k-1}\right)+\frac{1}{2} F_{2}(k) g\left(w_{2 k}\right)
\end{aligned}
$$

where $F_{i}(k), i=0,1,2$ and $k=2,3, \ldots, j$ are defined as above.

Together these estimates lead to (2.8) and the proof of Lemma 2.1 is complete.

Next we consider the discretisation of (2.5). At the node $t_{2 j+1}=\frac{2 j+1}{2 M}, j=1,2, \ldots, M-$ 1 we have

$$
\begin{aligned}
& { }_{0}^{R} D_{t}^{\alpha} y\left(t_{2 j+1}\right)=\frac{1}{\Gamma(-\alpha)} \oint_{0}^{t_{2 j+1}}\left(t_{2 j+1}-\tau\right)^{-1-\alpha} y(\tau) d \tau \\
& =\frac{1}{\Gamma(-\alpha)} \int_{0}^{t_{1}}\left(t_{2 j+1}-\tau\right)^{-1-\alpha} y(\tau) d \tau+\frac{t_{2 j+1}^{-\alpha}}{\Gamma(-\alpha)} \oint_{0}^{\frac{2 j}{2 j+1}} w^{-1-\alpha} y\left(t_{2 j+1}-t_{2 j+1} w\right) d w .
\end{aligned}
$$




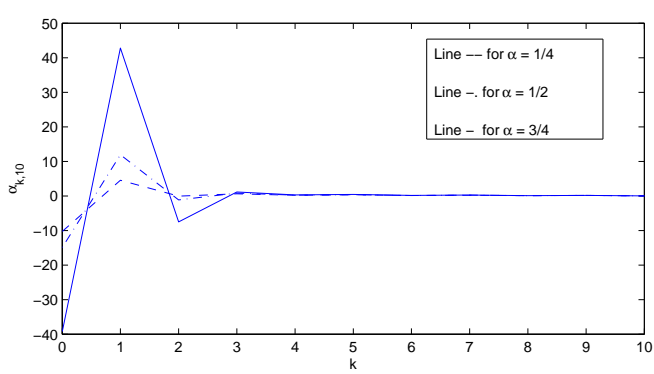

Fig. 2.1 The weights $\alpha_{k, 10}$ of the approximation of the fractional derivative with the different fractional order $\alpha$

For $j=1,2, \ldots, M-1$, we replace $g(w)=y\left(t_{2 j+1}-t_{2 j+1} w\right)$ in the integral in (2.11) by a piecewise quadratic interpolation polynomial with equispaced nodes $0, \frac{1}{2 j+1}, \frac{2}{2 j+1}, \ldots, \frac{2 j}{2 j+1}$. We then have, for any smooth function $g(w)$,

$$
\oint_{0}^{\frac{2 j}{2 j+1}} w^{-1-\alpha} g(w) d w=\oint_{0}^{\frac{2 j}{2 j+1}} w^{-1-\alpha} g_{2}(w) d w+R_{2 j+1}(g)
$$

where $g_{2}(w)$ is the piecewise quadratic interpolation polynomial of $g(w)$ with the nodes $0, \frac{1}{2 j+1}, \frac{2}{2 j+1}, \ldots, \frac{2 j}{2 j+1}$ and $R_{2 j+1}(g)$ is the remainder term.

Similarly we can prove the following lemma.

Lemma 2.2 Let $0<\alpha<1$. We have

$$
\oint_{0}^{\frac{2 j}{2 j+1}} w^{-1-\alpha} g_{2}(w) d w=\sum_{k=0}^{2 j} \alpha_{k, 2 j+1} g\left(\frac{k}{2 j+1}\right)
$$

where $\alpha_{k, 2 j+1}=\alpha_{k, 2 j}, k=0,1,2, \ldots, 2 j$ and $\alpha_{k, 2 j}$ are given in Lemma 2.1.

Remark 2.1 By the direct calculations, we can show that, with $0<\alpha<1$,

$$
\alpha_{0,2 j}=\frac{2^{-\alpha}(\alpha+2)}{(-\alpha)(-\alpha+1)(-\alpha+2)(2 j)^{-\alpha}}<0,
$$

and $\alpha_{k, 2 j}>0$ for $k>0, k \neq 2$. For $k=2$, there exists $\alpha_{1} \in(0,1)$ such that $\alpha_{2,2 j} \geq 0$ for $0<\alpha<\alpha_{1}$ and $\alpha_{2,2 j} \leq 0 \alpha_{1}<\alpha<1$.

To see the weights $\alpha_{k, 2 j}, k=0,1,2, \ldots, 2 j$, in Figure 2.1 , we plot $\alpha_{l, 10}$ for $l=$ $0,1,2, \ldots, 10$ with the different fractional order $\alpha=1 / 4,1 / 2,3 / 4$.

Now solutions of (2.1) satisfy, with $j=1,2, \ldots, M$,

$$
y\left(t_{2 j}\right)=\frac{1}{\alpha_{0,2 j}-t_{2 j}^{\alpha} \Gamma(-\alpha) \beta}\left[t_{2 j}^{\alpha} \Gamma(-\alpha) f\left(t_{2 j}\right)-\sum_{k=1}^{2 j} \alpha_{k, 2 j} y\left(t_{2 j-k}\right)+y_{0} \sum_{k=0}^{2 j} \alpha_{k, 2 j}-R_{2 j}(g)\right],
$$


and, with $j=1,2, \ldots, M-1$,

$$
\begin{aligned}
y\left(t_{2 j+1}\right) & =\frac{1}{\alpha_{0,2 j+1}-t_{2 j+1}^{\alpha} \Gamma(-\alpha) \beta}\left[t_{2 j+1}^{\alpha} \Gamma(-\alpha) f\left(t_{2 j+1}\right)-\sum_{k=1}^{2 j} \alpha_{k, 2 j+1} y\left(t_{2 j+1-k}\right)\right. \\
& \left.+y_{0} \sum_{k=0}^{2 j} \alpha_{k, 2 j+1}-R_{2 j+1}(g)-t_{2 j+1}^{\alpha} \int_{0}^{t_{1}}\left(t_{2 j+1}-\tau\right)^{-1-\alpha} y(\tau) d \tau\right] .
\end{aligned}
$$

Here $\alpha_{0, l}-t_{l}^{\alpha} \Gamma(-\alpha) \beta<0, l=2 j, 2 j+1$, which follow from (2.14) and $\Gamma(-\alpha)<$ $0, \beta<0$ and $\alpha_{0,2 j+1}=\alpha_{0,2 j}$.

Let $y_{2 j} \approx y\left(t_{2 j}\right)$ and $y_{2 j+1} \approx y\left(t_{2 j+1}\right)$ denote the approximations of the exact solutions $y\left(t_{2 j}\right)$ and $y\left(t_{2 j+1}\right)$, respectively. Assume that the starting values $y_{0}$ and $y_{1}$ are given. We define the following numerical methods for solving (2.1), with $j=1,2, \ldots, M$,

$$
y_{2 j}=\frac{1}{\alpha_{0,2 j}-t_{2 j}^{\alpha} \Gamma(-\alpha) \beta}\left[t_{2 j}^{\alpha} \Gamma(-\alpha) f\left(t_{2 j}\right)-\sum_{k=1}^{2 j} \alpha_{k, 2 j} y_{2 j-k}+y_{0} \sum_{k=0}^{2 j} \alpha_{k, 2 j}\right],
$$

and, with $j=1,2, \ldots, M-1$,

$$
\begin{aligned}
y_{2 j+1}= & \frac{1}{\alpha_{0,2 j+1}-t_{2 j+1}^{\alpha} \Gamma(-\alpha) \beta}\left[t_{2 j+1}^{\alpha} \Gamma(-\alpha) f\left(t_{2 j+1}\right)-\sum_{k=1}^{2 j} \alpha_{k, 2 j+1} y_{2 j+1-k}\right. \\
& \left.+y_{0} \sum_{k=0}^{2 j} \alpha_{k, 2 j+1}-t_{2 j+1}^{\alpha} \int_{0}^{t_{1}}\left(t_{2 j+1}-\tau\right)^{-1-\alpha} y(\tau) d \tau\right] .
\end{aligned}
$$

Remark 2.2 In practice, we need to approximate $\int_{0}^{t_{1}}\left(t_{2 j+1}-\tau\right)^{-1-\alpha} y(\tau) d \tau$. One way is to divide the integral $\left[0, t_{1}\right]$ into small intervals $0 \leq t_{1}^{1} \leq t_{1}^{2} \leq \cdots \leq t_{1}^{N}=t_{1}$ with stepsize $\tilde{h} \ll h$. We first obtain $y_{1 p} \approx y\left(t_{1}^{p}\right), p=1,2, \ldots, N$ by using some numerical methods for solving fractional differential equation. Then we apply a quadrature formula to approximate the integral.

We have the following asymptotic expansion theorem.

Theorem 2.1 Let $0<\alpha<1$ and $M$ be a positive integer. Let $0=t_{0}<t_{1}<t_{2}<$ $\cdots<t_{2 j}<t_{2 j+1}<\cdots<t_{2 M}=1$ be a partition of $[0,1]$ and $h$ the step size. Let $y\left(t_{2 j}\right), y\left(t_{2 j+1}\right), y_{2 j}$ and $y_{2 j+1}$ be the exact solutions and the approximate solutions of (2.15) - (2.18), respectively. Assume that the function $y \in C^{m+2}[0,1], m \geq 3$. Further assume that we obtain the exact starting values $y_{0}=y(0)$ and $y_{1}=y\left(t_{1}\right)$. Then there exist coefficients $c_{\mu}=c_{\mu}(\alpha)$ and $c_{\mu}^{*}=c_{\mu}^{*}(\alpha)$ such that the sequence $\left\{y_{l}\right\}, l=0,1,2, \ldots, 2 M$ possesses an asymptotic expansion of the form

$$
y\left(t_{2 M}\right)-y_{2 M}=\sum_{\mu=3}^{m+1} c_{\mu}(2 M)^{\alpha-\mu}+\sum_{\mu=2}^{\mu^{*}} c_{\mu}^{*}(2 M)^{-2 \mu}+o\left((2 M)^{\alpha-m-1}\right), \quad \text { for } M \rightarrow \infty,
$$

that is,

$$
y\left(t_{2 M}\right)-y_{2 M}=\sum_{\mu=3}^{m+1} c_{\mu} h^{\mu-\alpha}+\sum_{\mu=2}^{\mu^{*}} c_{\mu}^{*} h^{2 \mu}+o\left(h^{m+1-\alpha}\right), \quad \text { for } h \rightarrow 0,
$$


where $\mu^{*}$ is the integer satisfying $2 \mu^{*}<m+1-\alpha<2\left(\mu^{*}+1\right)$, and $c_{\mu}$ and $c_{\mu}^{*}$ are certain coefficients that depend on $y$.

To prove Theorem 2.1, we need the following lemma for the asymptotic expansions for the remainder terms $R_{2 j}(g)$ and $R_{2 j+1}(g)$ in (2.7) and (2.12).

Lemma 2.3 Let $0<\alpha<1$ and $g \in C^{m+2}[0,1], m \geq 3$. Let $R_{2 j}(g)$ and $R_{2 j+1}(g)$ be the remainder terms in (2.7) and (2.12), respectively. Then we have, with $l=$ $2,3, \ldots, 2 j, 2 j+1, \ldots, 2 M$,

$$
R_{l}(g)=\sum_{\mu=3}^{m+1} d_{\mu} l^{\alpha-\mu}+\sum_{\mu=2}^{\mu^{*}} d_{\mu}^{*} l^{-2 \mu}+o\left(l^{\alpha-m-1}\right),
$$

where $\mu^{*}$ is the integer satisfying $2 \mu^{*}<m+1-\alpha<2\left(\mu^{*}+1\right)$, and $d_{\mu}$ and $d_{\mu}^{*}$ are certain coefficients that depend on $g$.

Proof We follow the proof of Theorem 1.3 in [14] where the piecewise linear Lagrange interpolation polynomials are used.

We first consider the case $l=2 j$ for $j=1,2, \ldots, M$. Let $0=w_{0}<w_{1}<w_{2}<\cdots<$ $w_{2 j}=1, w_{k}=\frac{k}{2 j}, k=0,1,2, \ldots, 2 j$ be a partition of $[0,1]$. Let $h_{1}=\frac{1}{2 j}$ be the step size. Let $g_{2}(w)$ denote the piecewise quadratic Lagrange interpolation polynomial defined by $(2.9)$ on $\left[w_{2 l}, w_{2 l+2}\right], l=0,1,2, \ldots, j-1$. Then we have

$$
\begin{aligned}
& R_{2 j}(g)=\oint_{0}^{1} w^{-1-\alpha} g(w) d w-\oint_{0}^{1} w^{-1-\alpha} g_{2}(w) d w \\
& =\sum_{l=0}^{j-1} \int_{w_{2 l}}^{w_{2 l+2}} w^{-1-\alpha}\left(g(w)-g_{2}(w)\right) d w=\sum_{l=0}^{j-1} \int_{0}^{1}\left(w_{2 l}+2 h_{1} s\right)^{-1-\alpha}\left[g\left(w_{2 l}+2 h_{1} s\right)\right. \\
& \left.-\left(\frac{1}{2}(2 s-1)(2 s-2) g\left(w_{2 l}\right)-(2 s)(2 s-2) g\left(w_{2 l+1}\right)+\frac{1}{2}(2 s)(2 s-1) g\left(w_{2 l+2}\right)\right)\right]\left(2 h_{1}\right) d s .
\end{aligned}
$$

By using the Taylor formula, we have

$$
\begin{aligned}
& g\left(w_{2 l}\right)=g\left(w_{2 l}+2 h_{1} s\right)+\frac{g^{\prime}\left(w_{2 l}+2 h_{1} s\right)}{1 !}\left(-2 h_{1} s\right)+\frac{g^{\prime \prime}\left(w_{2 l}+2 h_{1} s\right)}{2 !}\left(-2 h_{1} s\right)^{2} \\
& +\frac{g^{\prime \prime \prime}\left(w_{2 l}+2 h_{1} s\right)}{3 !}\left(-2 h_{1} s\right)^{3}+\cdots+\frac{g^{(M)}\left(w_{2 l}+2 h_{1} s\right)}{m !}\left(-2 h_{1} s\right)^{m}+R_{m+1}^{(1)}, \\
& g\left(w_{2 l+1}\right)=g\left(w_{2 l}+2 h_{1} s\right)+\frac{g^{\prime}\left(w_{2 l}+2 h_{1} s\right)}{1 !}\left(h_{1}-2 h_{1} s\right)+\frac{g^{\prime \prime}\left(w_{2 l}+2 h_{1} s\right)}{2 !}\left(h_{1}-2 h_{1} s\right)^{2} \\
& +\frac{g^{\prime \prime \prime}\left(w_{2 l}+2 h_{1} s\right)}{3 !}\left(h_{1}-2 h_{1} s\right)^{3}+\cdots+\frac{g^{(m)}\left(w_{2 l}+2 h_{1} s\right)}{m !}\left(h_{1}-2 h_{1} s\right)^{m}+R_{m+1}^{(2)}, \\
& g\left(w_{2 l+2}\right)=g\left(w_{2 l}+2 h_{1} s\right)+\frac{g^{\prime}\left(w_{2 l}+2 h_{1} s\right)}{1 !}\left(2 h_{1}-2 h_{1} s\right)+\frac{g^{\prime \prime}\left(w_{2 l}+2 h_{1} s\right)}{2 !}\left(2 h_{1}-2 h_{1} s\right)^{2} \\
& +\frac{g^{\prime \prime \prime}\left(w_{2 l}+2 h_{1} s\right)}{3 !}\left(2 h_{1}-2 h_{1} s\right)^{3}+\cdots+\frac{g^{(m)}\left(w_{2 l}+2 h_{1} s\right)}{m !}\left(2 h_{1}-2 h_{1} s\right)^{m}+R_{m+1}^{(3)},
\end{aligned}
$$


where $R_{m+1}^{(i)}, i=1,2,3$ denote the remainder terms. Thus we obtain

$$
\begin{aligned}
R_{2 j}(g)= & \left(2 h_{1}\right) \sum_{l=0}^{j-1} \int_{0}^{1}\left(w_{2 l}+2 h_{1} s\right)^{-1-\alpha}\left[\sum_{r=0}^{m-3} h_{1}^{r+3} g^{(r+3)}\left(w_{2 l}+2 h_{1} s\right) \pi_{r}(s)\right] d s \\
& +\left(2 h_{1}\right) \sum_{l=0}^{j-1} \int_{0}^{1}\left(w_{2 l}+2 h_{1} s\right)^{-1-\alpha} \varepsilon_{m+1}(s) d s=I+I I,
\end{aligned}
$$

where $\varepsilon_{m+1}(s)$ depends on the remainder terms $R_{m+1}^{(i)}, i=1,2,3$ and $\pi_{r}(s)$ are some functions of $s$.

For $I$, we have

$$
I=\sum_{r=0}^{m-3} h_{1}^{r+3} \int_{0}^{1}\left[2 h_{1} \sum_{l=0}^{j-1}\left(w_{2 l}+2 h_{1} s\right)^{-1-\alpha} g^{(r+3)}\left(w_{2 l}+2 h_{1} s\right)\right] \pi_{r}(s) d s .
$$

Applying Theorem 3.2 in [20], we have, with $\bar{w}_{l}=w_{2 l}, \bar{h}_{1}=2 h_{1}$,

$$
\begin{aligned}
& 2 h_{1} \sum_{l=0}^{j-1}\left(w_{2 l}+2 h_{1} s\right)^{-1-\alpha} g^{(r+3)}\left(w_{2 l}+2 h_{1} s\right) \\
& =\bar{h}_{1} \sum_{l=0}^{j-1}\left(\bar{w}_{l}+\bar{h}_{1} s\right)^{-1-\alpha} g^{(r+3)}\left(\bar{w}_{l}+\bar{h}_{1} s\right) \\
& =\sum_{j=0}^{m-r-3} a_{j}(s) h_{1}^{j}+\sum_{j=0}^{m-r-2} a_{0, j}(s) h_{1}^{j-\alpha}+o\left(h_{1}^{m-r-2}\right),
\end{aligned}
$$

with some suitable functions $a_{j}(s), j=0,1, \ldots, m-r-3$ and $a_{0, j}(s), j=0,1, \ldots, m-$ $r-2$, with $r=0,1,2, \ldots, m-3, m \geq 3$.

Hence we have, noting that $h_{1}=(2 j)^{-1}$,

$$
\begin{aligned}
I & =\sum_{r=0}^{m-3} h_{1}^{3+r}\left[\int_{0}^{1} \sum_{j=0}^{m-r-3} a_{j}(s) h_{1}^{j} \pi_{r}(s) d s\right] \\
& \left.+\sum_{r=0}^{m-3} h_{1}^{3+r}\left[\int_{0}^{1} \sum_{j=0}^{m-r-2} a_{0, j}(s) h_{1}^{j-\alpha} \pi_{r}(s) d s\right)\right]+o\left(h_{1}^{m+1}\right) \\
& =\sum_{r=0}^{m-3} \sum_{j=0}^{m-r-3}\left[\int_{0}^{1} a_{j}(s) \pi_{r}(s) d s\right] h_{1}^{3+r+j} \\
& +\sum_{r=0}^{m-3} \sum_{j=0}^{m-r-2}\left[\int_{0}^{1} a_{0, j}(s) \pi_{r}(s) d s\right] h_{1}^{3+r+j-\alpha}+o\left(h_{1}^{m+1}\right) \\
& =\sum_{\mu=3}^{m+1} d_{\mu}(2 j)^{\alpha-\mu}+\sum_{\mu=2}^{\mu^{*}} d_{\mu}^{*}(2 j)^{-2 \mu}+o\left((2 j)^{-m-1}\right)
\end{aligned}
$$

where $\mu^{*}$ is the integer satisfying $2 \mu^{*}<m+1-\alpha<2\left(\mu^{*}+1\right)$, and $d_{\mu}$ and $d_{\mu}^{*}$ are certain coefficients that depend on $g$. We remark that the expansion does not contain 
any odd integer of powers of $(2 j)$ which follows from the argument in the proof of Theorem 1.3 in [14].

For II, we have, following the argument of the proof for Theorem 1.3 in [14],

$$
I I=2 h_{1} \sum_{l=0}^{j-1} \int_{0}^{1}\left(w_{2 l}+2 h_{1} s\right)^{-1-\alpha} \varepsilon_{m+1}(s) d s=o\left((2 j)^{\alpha-m-1}\right) .
$$

Thus (2.19) holds for $l=2 j$.

Next we consider the case $l=2 j+1$. Denote $w_{2 l}=\frac{2 l}{2 j+1}, w_{2 l+2}=\frac{2 l+2}{2 j+1}$ and $h_{1}=$ $\frac{1}{2 j+1}$, we have

$$
\begin{aligned}
& R_{2 j+1}(g)=\oint_{0}^{\frac{2 j}{2 j+1}} w^{-1-\alpha} g(w) d w-\oint_{0}^{\frac{2 j}{2 j+1}} w^{-1-\alpha} g_{2}(w) d w \\
& =\sum_{l=0}^{j-1} \int_{w_{2 l}}^{w_{2 l+2}} w^{-1-\alpha}\left(g(w)-g_{2}(w)\right) d w=\sum_{l=0}^{j-1} \int_{0}^{1}\left(w_{2 l}+2 h_{1} s\right)^{-1-\alpha}\left[g\left(w_{2 l}+2 h_{1} s\right)\right. \\
& \left.-\left(\frac{1}{2}(2 s-1)(2 s-2) g\left(w_{2 l}\right)-(2 s)(2 s-2) g\left(w_{2 l+1}\right)+\frac{1}{2}(2 s)(2 s-1) g\left(w_{2 l+2}\right)\right)\right]\left(2 h_{1}\right) d s .
\end{aligned}
$$

Following the same argument as for the case $l=2 j$, we show that (2.19) also holds for $l=2 j+1$. Together these estimates complete the proof of Lemma 2.3.

Proof (Proof of Theorem 2.1)

We follow the proof of Theorem 2.1 in [14] where the piecewise linear Lagrange interpolation polynomials are used to approximate the Hadamard finite-part integral.

Let us fix $t_{l}=c$ to be a constant for $l=1,2, \ldots 2 M$. We will investigate the difference

$$
e_{l}=y\left(t_{l}\right)-y_{l}, \quad \text { for } l \rightarrow \infty, \quad \text { with } t_{l}=l h=\frac{l}{2 M}=c,
$$

where $h=1 /(2 M)$ is the step size. In other words, there is a constant $c$, independent of $M$, such that

$$
l=c \cdot(2 M), \quad \text { or } M=l /(2 c),
$$

and consequently, we see that if $e_{l}$ possesses an asymptotic expansion w. r. t. $l$, then $e_{2 M}$ possesses at the same time one w. r. t. $M$, and vice versa.

We shall prove

$$
e_{l}=y\left(t_{l}\right)-y_{l}=\sum_{\mu=3}^{m+1} c_{\mu}(2 M)^{\alpha-\mu}+\sum_{\mu=2}^{\mu^{*}} c_{\mu}^{*}(2 M)^{-2 \mu}+o\left((2 M)^{\alpha-m-1}\right), \quad \text { for } l \rightarrow \infty
$$

for some suitable constants $c_{\mu}, c_{\mu}^{*}$ which we will determine later.

Let us first consider the case $l=2 j$. Subtracting (2.17) from (2.15), we have, noting $t_{2 j}=(2 j) h=\frac{2 j}{2 M}=c$,

$$
\begin{aligned}
e_{2 j} & =\frac{1}{\alpha_{0,2 j}-\left(\frac{2 j}{2 M}\right)^{\alpha} \Gamma(-\alpha) \beta}\left[-\sum_{k=1}^{2 j} \alpha_{k, 2 j}\left(y\left(t_{2 j-k}\right)-y_{2 j-k}\right)-R_{2 j}(g)\right] \\
& =\frac{1}{c^{\alpha} \Gamma(-\alpha) \beta-\alpha_{0,2 j}}\left(\sum_{k=1}^{2 j} \alpha_{k, 2 j} e_{2 j-k}+R_{2 j}(g)\right) .
\end{aligned}
$$


Note that $g(\cdot)=y\left(t_{2 j}-t_{2 j} \cdot\right) \in C^{m+2}[0,1], m \geq 3$, we have, by Lemma 2.3,

$$
R_{2 j}(g)=\sum_{\mu=3}^{m+1} d_{\mu}(2 j)^{\alpha-\mu}+\sum_{\mu=2}^{\mu^{*}} d_{\mu}^{*}(2 j)^{-2 \mu}+o\left((2 j)^{\alpha-m-1}\right), \quad \text { for } j \rightarrow \infty,
$$

where $\mu^{*}$ is the integer satisfying $2 \mu^{*}<m+1-\alpha<2\left(\mu^{*}+1\right)$, and $d_{\mu}$ and $d_{\mu}^{*}$ are certain coefficients that depend on $g$.

Note that $(2 j) /(2 M)=c$, we can write (2.24) into

$$
R_{2 j}(g)=\sum_{\mu=3}^{m+1} \tilde{d}_{\mu}(2 M)^{\alpha-\mu}+\sum_{\mu=2}^{\mu^{*}} \tilde{d}_{\mu}^{*}(2 M)^{-2 \mu}+o\left((2 M)^{\alpha-m-1}\right), \quad \text { for } j \rightarrow \infty .
$$

Choose

$$
\begin{aligned}
& c_{\mu}=\frac{1}{-c^{\alpha} \Gamma(-\alpha) \beta-1 / \alpha} \tilde{d}_{\mu}, \quad \mu=3,4, \ldots, m+1, \\
& c_{\mu}^{*}=\frac{1}{-c^{\alpha} \Gamma(-\alpha) \beta-1 / \alpha} \tilde{d}_{\mu}^{*}, \quad \mu=1,2, \ldots, \mu^{*},
\end{aligned}
$$

we will prove below that (2.22) holds for the coefficents $c_{\mu}, c_{\mu}^{*}$ defined in (2.26) and (2.27).

We shall use mathematical induction to prove (2.22). By assumption $e_{0}=0, e_{1}=$ 0 , hence (2.22) holds for $l=0,1$ with the coefficients given by (2.26) and (2.27). Let us now consider the case for $l=2$. We have, noting that $\alpha_{0, l}=\frac{2^{-\alpha}(\alpha+2)(2 M c)^{\alpha}}{(-\alpha)(-\alpha+1)(-\alpha+2)}$ and applying Lemma 2.3,

$$
\begin{aligned}
e_{2}= & y\left(t_{2}\right)-y_{2}=\frac{1}{c^{\alpha} \Gamma(-\alpha) \beta-\alpha_{0,2}}\left(\sum_{k=1}^{2} \alpha_{k, 2} e_{2-k}+R_{2}(g)\right) \\
= & \frac{1}{c^{\alpha} \Gamma(-\alpha) \beta-\alpha_{0,2}}\left[\left(\sum_{\mu=3}^{m+1} c_{\mu}(2 M)^{\alpha-\mu}+\sum_{\mu=2}^{\mu^{*}} c_{\mu}^{*}(2 M)^{-2 \mu}+o\left((2 M)^{\alpha-m-1}\right)\right)\right. \\
& \left.\cdot\left(\sum_{k=0}^{2} \alpha_{k, 2}-\alpha_{0,2}\right)+R_{2}(g)\right] .
\end{aligned}
$$

Thus we get, noting that $\sum_{k=0}^{2} \alpha_{k, 2}=-1 / \alpha$ and $\alpha_{0,2}=\frac{2^{-\alpha}(\alpha+2)(2 M c)^{\alpha}}{(-\alpha)(-\alpha+1)(-\alpha+2)}$,

$$
\begin{aligned}
& {\left[\frac{2^{-\alpha}(\alpha+2)(2 M c)^{\alpha}}{(-\alpha)(-\alpha+1)(-\alpha+2)}-c^{\alpha} \Gamma(-\alpha) \beta\right] e_{2}} \\
& =\frac{1}{\alpha}\left[\sum_{\mu=3}^{m+1} c_{\mu}(2 M)^{\alpha-\mu}+\sum_{\mu=2}^{\mu^{*}} c_{\mu}^{*}(2 M)^{-2 \mu}+o\left((2 M)^{\alpha-m-1}\right)\right] \\
& -\sum_{\mu=3}^{m+1} \tilde{d}_{\mu}(2 M)^{\alpha-\mu}-\sum_{\mu=2}^{\mu^{*}} \tilde{d}_{\mu}^{*}(2 M)^{-2 \mu}+o\left((2 M)^{\alpha-m-1}\right) \\
& +\frac{2^{-\alpha}(\alpha+2)(2 M c)^{\alpha}}{(-\alpha)(-\alpha+1)(-\alpha+2)}\left[\sum_{\mu=3}^{m+1} c_{\mu}(2 M)^{\alpha-\mu}+\sum_{\mu=2}^{\mu^{*}} c_{\mu}^{*}(2 M)^{-2 \mu}+o\left((2 M)^{\alpha-m-1}\right)\right]
\end{aligned}
$$


This shows that the sequence $e_{2}$ possesses an asymptotic expansion with respect to the powers of $2 M$, and it is easy to check that, by comparing with the coefficients of powers of $(2 M)$, see [14],

$$
e_{2}=\sum_{\mu=3}^{m+1} c_{\mu}(2 M)^{\alpha-\mu}+\sum_{\mu=2}^{\mu^{*}} c_{\mu}^{*}(2 M)^{-2 \mu}+o\left((2 M)^{\alpha-m-1}\right) .
$$

Assume that (2.22) holds for $l=0,1, \ldots, 2 j-1$. Then we have, following the same argument for (2.29), noting $\sum_{k=0}^{2 j} \alpha_{k, 2 j}=-1 / \alpha$ and applying Lemma 2.3,

$$
\begin{aligned}
& {\left[\frac{2^{-\alpha}(\alpha+2)(2 M c)^{\alpha}}{(-\alpha)(-\alpha+1)(-\alpha+2)}-c^{\alpha} \Gamma(-\alpha) \beta\right] e_{2 j}} \\
& =\frac{1}{\alpha}\left[\sum_{\mu=3}^{m+1} c_{\mu}(2 M)^{\alpha-\mu}+\sum_{\mu=2}^{\mu^{*}} c_{\mu}^{*}(2 M)^{-2 \mu}+o\left((2 M)^{\alpha-m-1}\right)\right] \\
& -\sum_{\mu=3}^{m+1} \tilde{d}_{\mu}(2 M)^{\alpha-\mu}-\sum_{\mu=2}^{\mu^{*}} \tilde{d}_{\mu}^{*}(2 M)^{-2 \mu}+o\left((2 M)^{\alpha-m-1}\right) \\
& +\frac{2^{-\alpha}(\alpha+2)(2 M c)^{\alpha}}{(-\alpha)(-\alpha+1)(-\alpha+2)}\left[\sum_{\mu=3}^{m+1} c_{\mu}(2 M)^{\alpha-\mu}+\sum_{\mu=2}^{\mu^{*}} c_{\mu}^{*}(2 M)^{-2 \mu}+o\left((2 M)^{\alpha-m-1}\right)\right]
\end{aligned}
$$

This shows that the sequence $e_{2 j}$ possesses an asymptotic expansion with respect to the powers of $2 M$, and it is easy to check that, by comparing with the coefficients of powers of $(2 M)$, see [14],

$$
e_{2 j}=\sum_{\mu=3}^{m+1} c_{\mu}(2 M)^{\alpha-\mu}+\sum_{\mu=2}^{\mu^{*}} c_{\mu}^{*}(2 M)^{-2 \mu}+o\left((2 M)^{\alpha-m-1}\right) .
$$

Hence (2.22) holds for $l=2 j$.

Finally we assume that (2.22) holds for $l=0,1, \ldots, 2 j$. Then we have, following the same argument for (2.30), noting $\sum_{k=0}^{2 j} \alpha_{k, 2 j+1}=\sum_{k=0}^{2 j} \alpha_{k, 2 j}=-1 / \alpha, \alpha_{0,2 j+1}=$ $\alpha_{0,2 j}$ and applying Lemma 2.3,

$$
\begin{aligned}
& {\left[\frac{2^{-\alpha}(\alpha+2)(2 M c)^{\alpha}}{(-\alpha)(-\alpha+1)(-\alpha+2)}-c^{\alpha} \Gamma(-\alpha) \beta\right] e_{2 j+1}} \\
& =\frac{1}{\alpha}\left[\sum_{\mu=3}^{m+1} c_{\mu}(2 M)^{\alpha-\mu}+\sum_{\mu=2}^{\mu^{*}} c_{\mu}^{*}(2 M)^{-2 \mu}+o\left((2 M)^{\alpha-m-1}\right)\right] \\
& -\sum_{\mu=3}^{m+1} \tilde{d}_{\mu}(2 M)^{\alpha-\mu}-\sum_{\mu=2}^{\mu^{*}} \tilde{d}_{\mu}^{*}(2 M)^{-2 \mu}+o\left((2 M)^{\alpha-m-1}\right) \\
& +\frac{2^{-\alpha}(\alpha+2)(2 M c)^{\alpha}}{(-\alpha)(-\alpha+1)(-\alpha+2)}\left[\sum_{\mu=3}^{m+1} c_{\mu}(2 M)^{\alpha-\mu}+\sum_{\mu=2}^{\mu^{*}} c_{\mu}^{*}(2 M)^{-2 \mu}+o\left((2 M)^{\alpha-m-1}\right)\right]
\end{aligned}
$$


This again shows that the sequence $e_{2 j+1}$ possesses an asymptotic expansion with respect to the powers of $2 M$, and it is easy to check that, by comparing with the coefficients of powers of $2 M$, see [14],

$$
e_{2 j+1}=\sum_{\mu=3}^{m+1} c_{\mu}(2 M)^{\alpha-\mu}+\sum_{\mu=2}^{\mu^{*}} c_{\mu}^{*}(2 M)^{-2 \mu}+o\left((2 M)^{\alpha-m-1}\right) .
$$

Hence (2.22) holds also for $l=2 j+1$. Together these estimates complete the proof of (2.22). Applying $l=2 M$ in (2.22), we get (2.23). The proof of Theorem 2.1 is now complete.

Remark 2.3 In Theorem 2.1, we assume that $y_{1}=y\left(t_{1}\right)$ exactly. In practice $y_{1}$ can be approximated by using the ideas described in Remark 2.2.

\section{A higher order fractional Adams-type method}

In this section we will consider a higher order numerical method for solving (1.1)(1.2). For simplicity we only consider the case where $0<\alpha \leq 2$ since the case $\alpha>2$ does not seem to be of major practical interest [11].

To make sure that (1.1) - (1.2) has a unique solution, we assume that $f(u, \cdot)$ satisfies a Lipschitz condition, i.e., there exists a constant $L$ such that

$$
|f(u, x)-f(u, y)| \leq L|x-y|, \quad \forall x, y \in \mathbf{R} .
$$

Let $m$ be a positive integer and let $0=t_{0}<t_{1}<t_{2}<\cdots<t_{2 j}<t_{2 j+1}<\cdots<$ $t_{2 m}=T$ be a partition of $[0, T]$ and $h$ the stepsize. Note that the system (1.1)-(1.2) is equivalent to (1.5). Let us now consider the discretisation of (1.5). At node $t=$ $t_{2 j}, j=1,2, \ldots, m$, we have

$$
y\left(t_{2 j}\right)=y_{0}+y_{0}^{(1)} \frac{t_{2 j}}{1 !}+\frac{1}{\Gamma(\alpha)} \int_{0}^{t_{2 j}}\left(t_{2 j}-u\right)^{\alpha-1} f(u, y(u)) d u
$$

(The second of the initial conditions only for $1<\alpha<2$ of course). At node $t=$ $t_{2 j+1}, j=1,2, \ldots, m-1$, we have

$$
\begin{aligned}
y\left(t_{2 j+1}\right)= & y_{0}+y_{0}^{(1)} \frac{t_{2 j+1}}{1 !}+\frac{1}{\Gamma(\alpha)} \int_{0}^{t_{2 j+1}}\left(t_{2 j+1}-u\right)^{\alpha-1} f(u, y(u)) d u \\
= & y_{0}+y_{0}^{(1)} \frac{t_{2 j+1}}{1 !}+\frac{1}{\Gamma(\alpha)} \int_{0}^{t_{1}}\left(t_{2 j+1}-u\right)^{\alpha-1} f(u, y(u)) d u \\
& +\frac{1}{\Gamma(\alpha)} \int_{t_{1}}^{t_{2 j+1}}\left(t_{2 j+1}-u\right)^{\alpha-1} f(u, y(u)) d u \\
= & y_{0}+y_{0}^{(1)} \frac{t_{2 j+1}}{1 !}+\frac{1}{\Gamma(\alpha)} \int_{0}^{t_{1}}\left(t_{2 j+1}-u\right)^{\alpha-1} f(u, y(u)) d u \\
& +\frac{1}{\Gamma(\alpha)} \int_{0}^{t_{2 j}}\left(t_{2 j}-u\right)^{\alpha-1} f(u+h, y(u+h)) d u
\end{aligned}
$$


We will replace $f(u, y(u))$ in the integral $\int_{0}^{t_{2 j}}\left(t_{2 j}-u\right)^{\alpha-1} f(u, y(u)) d u$ in (3.2) by the following piecewise quadratic polynomial, for $t_{2 l} \leq u \leq t_{2 l+2}, l=0,1,2, \ldots j-1$ with $j=1,2, \ldots, m$,

$$
\begin{aligned}
f(u, y(u)) \approx P_{2}(u)= & \frac{\left(u-t_{2 l+1}\right)\left(u-t_{2 l+2}\right)}{\left(t_{2 l}-t_{2 l+1}\right)\left(t_{2 l}-t_{2 l+2}\right)} f\left(t_{2 l}, y\left(t_{2 l}\right)\right) \\
& +\frac{\left(u-t_{2 l}\right)\left(u-t_{2 l+2}\right)}{\left(t_{2 l+1}-t_{2 l}\right)\left(t_{2 l+1}-t_{2 l+2}\right)} f\left(t_{2 l+1}, y\left(t_{2 l+1}\right)\right) \\
& +\frac{\left(u-t_{2 l}\right)\left(u-t_{2 l+1}\right)}{\left(t_{2 l+2}-t_{2 l}\right)\left(t_{2 l+2}-t_{2 l+1}\right)} f\left(t_{2 l+2}, y\left(t_{2 l+2}\right)\right) .
\end{aligned}
$$

Similarly we will replace $f(u+h, f(u+h))$ in the integral $\int_{0}^{t_{2 j}}\left(t_{2 j}-u\right)^{\alpha-1} f(u+$ $h, y(u+h)) d u$ in (3.3) by the following piecewise quadratic polynomial, for $t_{2 l} \leq$ $u \leq t_{2 l+2}, l=0,1,2, \ldots j-1, j=1,2, \ldots, m-1$,

$$
\begin{aligned}
f(u+h, y(u+h)) \approx Q_{2}(u)= & \frac{\left(u-t_{2 l+1}\right)\left(u-t_{2 l+2}\right)}{\left(t_{2 l}-t_{2 l+1}\right)\left(t_{2 l}-t_{2 l+2}\right)} f\left(t_{2 l+1}, y\left(t_{2 l+1}\right)\right) \\
& +\frac{\left(u-t_{2 l}\right)\left(u-t_{2 l+2}\right)}{\left(t_{2 l+1}-t_{2 l}\right)\left(t_{2 l+1}-t_{2 l+2}\right)} f\left(t_{2 l+2}, y\left(t_{2 l+2}\right)\right) \\
& +\frac{\left(u-t_{2 l}\right)\left(u-t_{2 l+1}\right)}{\left(t_{2 l+2}-t_{2 l}\right)\left(t_{2 l+2}-t_{2 l+1}\right)} f\left(t_{2 l+3}, y\left(t_{2 l+3}\right)\right) .
\end{aligned}
$$

We then have the following lemma:

Lemma 3.1 Let $0<\alpha \leq 2$. We have

$$
\int_{0}^{t_{2 j}}\left(t_{2 j}-u\right)^{\alpha-1} P_{2}(u) d u=\sum_{k=0}^{2 j} c_{k, 2 j} f\left(t_{k}, y\left(t_{k}\right)\right),
$$

and

$$
\int_{0}^{t_{2 j}}\left(t_{2 j}-u\right)^{\alpha-1} Q_{2}(u) d u=\sum_{k=0}^{2 j} c_{k, 2 j} f\left(t_{k+1}, y\left(t_{k+1}\right)\right)
$$

where

$$
c_{k, 2 j}=\frac{h^{\alpha}}{\alpha(\alpha+1)(\alpha+2)}\left\{\begin{array}{l}
\frac{1}{2} F_{0}(0), \quad \text { if } k=0, \\
\frac{1}{2} F_{0}(l)+\frac{1}{2} F_{2}(l-1), \quad \text { if } k=2 l, l=1,2, \ldots, j-1, \\
-F_{1}(l), \quad \text { if } k=2 l+1, l=0,1,2, \ldots, j-1, \\
\frac{1}{2} F_{2}(j-1), \quad \text { if } k=2 j,
\end{array}\right.
$$


and

$$
\begin{aligned}
F_{0}(l)= & \alpha(\alpha+1)\left((2 j-2 l)^{\alpha+2}-(2 j-2 l-2)^{\alpha+2}\right) \\
& +\alpha(\alpha+2)(2(2 j)-(2 l+1)-(2 l+2))\left((2 j-2 l-2)^{\alpha+1}-(2 j-2 l)^{\alpha+1}\right) \\
& +(\alpha+1)(\alpha+2)((2 j-2 l-1)(2 j-2 l-2))\left((2 j-2 l)^{\alpha}-(2 j-2 l-2)^{\alpha}\right), \\
F_{1}(l)= & \alpha(\alpha+1)\left((2 j-2 l)^{\alpha+2}-(2 j-2 l-2)^{\alpha+2}\right) \\
& +\alpha(\alpha+2)(2(2 j)-(2 l)-(2 l+2))\left((2 j-2 l-2)^{\alpha+1}-(2 j-2 l)^{\alpha+1}\right) \\
& +(\alpha+1)(\alpha+2)((2 j-2 l)(2 j-2 l-2))\left((2 j-2 l)^{\alpha}-(2 j-2 l-2)^{\alpha}\right), \\
F_{2}(l)= & \alpha(\alpha+1)\left((2 j-2 l)^{\alpha+2}-(2 j-2 l-2)^{\alpha+2}\right) \\
& +\alpha(\alpha+2)(2(2 j)-(2 l)-(2 l+1))\left((2 j-2 l-2)^{\alpha+1}-(2 j-2 l)^{\alpha+1}\right) \\
& +(\alpha+1)(\alpha+2)((2 j-2 l)(2 j-2 l-1))\left((2 j-2 l)^{\alpha}-(2 j-2 l-2)^{\alpha}\right),
\end{aligned}
$$

Proof This follows from a simple calculation. We omit the proof here.

We now define a fractional Adams numerical method for solving (1.5). Let $y_{l} \approx$ $y\left(t_{l}\right)$ denote the approximation of $y\left(t_{l}\right), l=0,1,2, \ldots, 2 m$. The corrector formula is defined by

$$
y_{2 j}=y_{0}+y_{0}^{(1)} \frac{t_{2 j}}{1 !}+\frac{1}{\Gamma(\alpha)}\left(\sum_{k=0}^{2 j-1} c_{k, 2 j} f\left(t_{k}, y_{k}\right)+c_{2 j, 2 j} f\left(t_{2 j}, y_{2 j}^{P}\right)\right), \quad j=1,2, \ldots, m,
$$

and

$$
\begin{aligned}
y_{2 j+1}= & y_{0}+y_{0}^{(1)} \frac{t_{2 j+1}}{1 !}+\frac{1}{\Gamma(\alpha)} \int_{0}^{t_{1}}\left(t_{2 j+1}-u\right)^{\alpha-1} f(u, y(u)) d u \\
& +\frac{1}{\Gamma(\alpha)}\left(\sum_{k=0}^{2 j-1} c_{k, 2 j} f\left(t_{k+1}, y_{k+1}\right)+c_{2 j, 2 j} f\left(t_{2 j+1}, y_{2 j+1}^{P}\right)\right), \quad j=1,2, \ldots, m-1 .
\end{aligned}
$$

The remaining problem is the determination of the predictor formula required to calculate $y_{k}^{P}$. The idea is the same as the one described above: we replace $f(u, y(u))$ and $f(u+h, y(u+h))$ of the integrals on the right-hand sides of equations (3.2) and (3.3), respectively, by the piecewise linear interpolation polynomials and obtain

$$
y_{2 j}^{P}=y_{0}+y_{0}^{(1)} \frac{t_{2 j}}{1 !}+\frac{1}{\Gamma(\alpha)}\left(\sum_{k=0}^{2 j-1} a_{k, 2 j} f\left(t_{k}, y_{k}\right)+a_{2 j, 2 j} f\left(t_{2 j}, y_{2 j}^{P P}\right)\right), \quad j=1,2, \ldots, m,
$$

and, with $j=1,2, \ldots, m-1$,

$$
y_{2 j+1}^{P}=y_{0}+y_{0}^{(1)} \frac{t_{2 j+1}}{1 !}+\frac{1}{\Gamma(\alpha)}\left(\sum_{k=0}^{2 j} a_{k, 2 j+1} f\left(t_{k}, y_{k}\right)+a_{2 j+1,2 j+1} f\left(t_{2 j+1}, y_{2 j+1}^{P P}\right)\right) \text {, }
$$


where the weights are [11]

$a_{k, n+1}=\frac{h^{\alpha}}{\alpha(\alpha+1)}\left\{\begin{array}{l}n^{\alpha+1}-(n-\alpha)(n+1)^{\alpha}, \quad \text { if } k=0, \\ (n-k+2)^{\alpha+1}+(n-k)^{\alpha+1}-2(n-k+1)^{\alpha+1} \quad \text { if } 1 \leq k \leq n, \\ 1, \quad \text { if } k=n+1 .\end{array}\right.$

Similarly, to calculate $y_{k}^{P P}$, we replace $f(u, y(u))$ and $f(u+h, y(u+h))$ in the integrals on the right-hand sides of equations (3.2) and (3.3), respectively, by the piecewise constants and obtain

$$
y_{2 j}^{P P}=y_{0}+y_{0}^{(1)} \frac{t_{2 j}}{1 !}+\frac{1}{\Gamma(\alpha)} \sum_{k=0}^{2 j-1} b_{k, 2 j} f\left(t_{k}, y_{k}\right), \quad j=1,2, \ldots, m,
$$

and

$$
y_{2 j+1}^{P P}=y_{0}+y_{0}^{(1)} \frac{t_{2 j+1}}{1 !}+\frac{1}{\Gamma(\alpha)} \sum_{k=0}^{2 j} b_{k, 2 j+1} f\left(t_{k}, y_{k}\right), \quad j=1,2, \ldots, m-1 .
$$

where the weights [11]

$$
b_{k, n+1}=\frac{h^{\alpha}}{\alpha}\left((n+1-k)^{\alpha}-(n-k)^{\alpha}\right) .
$$

Our basic fractional Adams method, is completely described now by equations (3.8) - (3.13).

Remark 3.1 In practice, we need to approximate the integral in (3.9). We shall use the same ideas as in Remark 2.2.

We have thus completed the description of our numerical algorithm. The remainder of the paper will be devoted to the error analysis of the scheme.

We have the following theorem.

Theorem 3.1 Let $0<\alpha \leq 2$ and assume that ${ }_{0}^{C} D_{t}^{\alpha} y \in C^{3}[0, T]$ for some suitable chosen T. Let $y\left(t_{k}\right)$ and $y_{k}, k=0,1,2, \ldots, 2 m, t_{2 m}=T$ be the solutions of (3.2), (3.3), (3.8), (3.9), respectively. Assume that $y_{0}=y(0)$ and $y_{1}=y\left(t_{1}\right)$ exactly. Then there exists a positive constant $C_{0}>0$ such that

$$
\max _{0 \leq k \leq 2 m}\left|y\left(t_{k}\right)-y_{k}\right| \leq\left\{\begin{array}{l}
C_{0} h^{1+2 \alpha}, \quad \text { if } 0<\alpha \leq 1 \\
C_{0} h^{3}, \quad \text { if } 1<\alpha \leq 2
\end{array}\right.
$$

To prove this theorem, we need some lemmas.

Lemma 3.2 ( Theorem 2.4 [11]) Let $0<\alpha \leq 2$. If $z \in C^{1}[0, T]$, then there is a constant $C_{1}^{\alpha}$ depending only on $\alpha$ such that

$$
\left|\int_{0}^{t_{2 j}}\left(t_{2 j}-u\right)^{\alpha-1} z(u) d u-\sum_{k=0}^{2 j-1} b_{k, 2 j} z\left(t_{k}\right)\right| \leq C_{1}^{\alpha} t_{2 j}^{\alpha} h .
$$


Lemma 3.3 ( Theorem 2.5 [11]) Let $0<\alpha \leq 2$. If $z \in C^{2}[0, T]$, then there is a constant $C_{2}^{\alpha}$ depending only on $\alpha$ such that

$$
\left|\int_{0}^{t_{2 j}}\left(t_{2 j}-u\right)^{\alpha-1} z(u) d u-\sum_{k=0}^{2 j} a_{k, 2 j} z\left(t_{k}\right)\right| \leq C_{2}^{\alpha} t_{2 j}^{\alpha} h^{2} .
$$

Lemma 3.4 Let $0<\alpha \leq 2$. If $z \in C^{3}[0, T]$, then there is a constant $C_{3}^{\alpha}$ depending only on $\alpha$ such that

$$
\left|\int_{0}^{t_{2 j}}\left(t_{2 j}-u\right)^{\alpha-1} z(u) d u-\sum_{k=0}^{2 j} c_{k, 2 j} z\left(t_{k}\right)\right| \leq C_{3}^{\alpha} t_{2 j}^{\alpha} h^{3} .
$$

and

$$
\left|\int_{t_{1}}^{t_{2 j+1}}\left(t_{2 j+1}-u\right)^{\alpha-1} z(u) d u-\sum_{k=0}^{2 j} c_{k, 2 j} z\left(t_{k+1}\right)\right| \leq C_{3}^{\alpha} t_{2 j+1}^{\alpha} h^{3} .
$$

Proof We have

$$
\begin{aligned}
I & =\int_{0}^{t_{2 j}}\left(t_{2 j}-u\right)^{\alpha-1} z(u) d u-\sum_{k=0}^{2 j} c_{k, 2 j} z\left(t_{k}\right) \\
& =\int_{0}^{t_{2 j}}\left(t_{2 j}-u\right)^{\alpha-1} z(u) d u-\int_{0}^{t_{2 j}}\left(t_{2 j}-u\right)^{\alpha-1} P_{2}(u) d u
\end{aligned}
$$

where $P_{2}(u)$ is the piecewise quadratic interpolation polynomial of $z(u)$, defined by (3.4).

Thus we have

$$
\begin{aligned}
|I| & =\left|\sum_{k=0}^{j-1} \int_{t_{2 k}}^{t_{2 k+2}}\left(t_{2 j}-u\right)^{\alpha-1}\left(z(u)-P_{2}(u)\right) d u\right| \\
& =\left|\sum_{k=0}^{j-1} \int_{t_{2 k}}^{t_{2 k+2}}\left(t_{2 j}-u\right)^{\alpha-1} \frac{z^{\prime \prime \prime}(\xi)}{3 !}\left(u-t_{2 k}\right)\left(u-t_{2 k+1}\right)\left(u-t_{2 k+2}\right) d u\right| \\
& \leq \frac{\left\|f^{\prime \prime \prime}\right\|_{\infty}}{3 !}(2 h)^{3} \int_{0}^{t_{2 j}}\left(t_{2 j}-u\right)^{\alpha-1} d u=C_{3}^{\alpha} t_{2 j}^{\alpha} h^{3},
\end{aligned}
$$

which shows (3.15). Similarly we can show (3.16).

Lemma 3.5 [11] Let $0<\alpha \leq 2$ and $m$ and $n$ be positive integers. Let $a_{k, 2 j}$ and $b_{k, 2 j}, k=0,1,2, \ldots, 2 j, j=1,2, \ldots, m$ be introduced in (3.10) and (3.12), respectively. Then we have

$$
a_{k, 2 j} \geq 0, \quad b_{k, 2 j} \geq 0, \quad k=0,1,2, \ldots 2 j,
$$

and

$$
\sum_{k=0}^{2 j} a_{k, 2 j} \leq \frac{1}{\alpha} T^{\alpha}, \quad \sum_{k=0}^{2 j} b_{k, 2 j} \leq \frac{1}{\alpha} T^{\alpha} . \quad j=1,2, \ldots, m .
$$

Further, there exist constants $D_{1}^{\alpha}$ and $D_{2}^{\alpha}$ such that

$$
a_{2 j, 2 j}=D_{2}^{\alpha} h^{\alpha}, \quad b_{2 j, 2 j}=D_{1}^{\alpha} h^{\alpha}, \quad j=1,2, \ldots, m .
$$


Lemma 3.6 Let $0<\alpha \leq 2$. Let $c_{k, 2 j}, k=0,1,2, \ldots, 2 j, j=1,2, \ldots$, m be introduced in (3.8). Then we have

$$
c_{k, 2 j} \geq 0, \quad k=0,1,2, \ldots, 2 j
$$

and

$$
\sum_{k=0}^{2 j} c_{k, 2 j} \leq \frac{1}{\alpha} T^{\alpha}
$$

Further there exists a constant $D_{3}^{\alpha}$ such that

$$
c_{2 j, 2 j}=D_{3}^{\alpha} h^{\alpha}, j=1,2, \ldots, m .
$$

Proof We first show that

$$
F_{1}(l) \leq 0, \quad l=0,1,2, \ldots, j-1 .
$$

It is easy to show that

$$
\begin{aligned}
F_{1}(l)= & 2\left((2 j-2 l)^{\alpha+2}-(\alpha+2)(2 j-2 l)^{\alpha+1}-(2 j-2 l-2)^{\alpha+2}\right. \\
& \left.-(\alpha+2)(2 j-2 l-2)^{\alpha+1}\right), \quad l=0,1,2, \ldots, j-1 .
\end{aligned}
$$

Further, after some direct calculations, we can show that

$$
(\gamma+1)(n+2)^{\gamma}+(\gamma+1) n^{\gamma}+n^{\gamma+1}-(n+2)^{\gamma+1} \geq 0, \quad \forall n \in \mathbf{Z}^{+}, \gamma>0 .
$$

By putting $n=2 j-2 l-2$ and $\gamma=\alpha+1$, we get (3.21).

Next we show

$$
F_{0}(l)+F_{2}(l-1) \geq 0, \quad l=1,2, \ldots, j-1 .
$$

It is easy to show that

$$
\begin{aligned}
F_{0}(l)+F_{2}(l-1)= & 2(2 j-2 l+2)^{\alpha+2}-(\alpha+2)(2 j-2 l+2)^{\alpha+1}-6(\alpha+2)(2 j-2 l)^{\alpha+1} \\
& -2(2 j-2 l-2)^{\alpha+2}-(\alpha+2)(2 j-2 l-2)^{\alpha+1} .
\end{aligned}
$$

Further, after some direct calculations, we can show that

$2(n+4)^{\alpha+2}-(\alpha+2)(n+4)^{\alpha+1}-6(\alpha+2)(n+2)^{\alpha+1}-2 n^{\alpha+2}-(\alpha+2) n^{\alpha+1} \geq 0, \quad \forall n \in \mathbf{Z}^{+}$.

Hence (3.22) follows from (3.23). Finally we can also show $F_{0}(0) \geq 0$ and $F_{2}(j-1) \geq$ 0 . Therefore we get (3.18).

Further (3.19) follows from

$$
\sum_{k=0}^{2 j} c_{k, 2 j}=\int_{0}^{t_{2 j}}\left(t_{2 j}-u\right)^{\alpha-1} d u=\frac{1}{\alpha} t_{2 j}^{\alpha} \leq \frac{1}{\alpha} T^{\alpha} .
$$

For (3.20), we have, by Lemma 3.1, $c_{2 j, 2 j}=\frac{1}{2} F_{2}(j-1)=D_{3}^{\alpha} h^{\alpha}$, with the suitable constant $D_{3}^{\alpha}$. Together these estimates complete the proof of Lemma 3.6. 
Proof (Proof of Theorem 3.1) We first consider the case where $1<\alpha \leq 2$. We will use mathematical induction. Note that, by assumptions, $\left|y\left(t_{0}\right)-y_{0}\right|=0,\left|y\left(t_{1}\right)-y_{1}\right|=0$. Assume that

$$
\left|y\left(t_{k}\right)-y_{k}\right| \leq C_{0} h^{3}
$$

is true for $k=0,1,2, \ldots, 2 j-1, j=1,2, \ldots, m$. We must prove that this also holds for $k=2 j$. In fact, we have, with $j=1,2, \ldots, m$,

$$
\begin{aligned}
& \Gamma(\alpha)\left(y\left(t_{2 j}\right)-y_{2 j}\right) \\
= & \int_{0}^{t_{2 j}}\left(t_{2 j}-u\right)^{\alpha-1} f(u, y(u)) d u-\left(\sum_{k=0}^{2 j-1} c_{k, 2 j} f\left(t_{k}, y_{k}\right)-c_{2 j, 2 j} f\left(t_{2 j}, t_{2 j}^{P}\right)\right) \\
= & \int_{0}^{t_{2 j}}\left(t_{2 j}-u\right)^{\alpha-1} f(u, y(u)) d u-\int_{0}^{t_{2 j}}\left(t_{2 j}-u\right)^{\alpha-1} P_{2}(u) d u \\
& +\int_{0}^{t_{2 j}}\left(t_{2 j}-u\right)^{\alpha-1} P_{2}(u) d u-\left(\sum_{k=0}^{2 j-1} c_{k, 2 j} f\left(t_{k}, y_{k}\right)-c_{2 j, 2 j} f\left(t_{2 j}, t_{2 j}^{P}\right)\right) \\
= & \left(\int_{0}^{t_{2 j}}\left(t_{2 j}-u\right)^{\alpha-1} f(u, y(u)) d u-\int_{0}^{t_{2 j}}\left(t_{2 j}-u\right)^{\alpha-1} P_{2}(u) d u\right) \\
& +\sum_{k=0}^{2 j-1} c_{k, 2 j}\left(f\left(t_{k}, y\left(t_{k}\right)\right)-f\left(t_{k}, y_{k}\right)\right)+c_{2 j, 2 j}\left(f\left(t_{2 j}, y\left(t_{2 j}\right)\right)-f\left(t_{2 j}, t_{2 j}^{P}\right)\right) \\
= & I_{1}+I I_{1}+I I I_{1} .
\end{aligned}
$$

For $I_{1}$, we have, by Lemma 3.4,

$\left|I_{1}\right|=\left|\int_{0}^{t_{2 j}}\left(t_{2 j}-u\right)^{\alpha-1} f(u, y(u)) d u-\int_{0}^{t_{2 j}}\left(t_{2 j}-u\right)^{\alpha-1} P_{2}(u) d u\right| \leq C_{3}^{\alpha} T^{\alpha} h^{3}$.

For $I I_{1}$, we have, by Lemma 3.6 and the Lipschitz condition (3.1),

$$
\begin{aligned}
\left|I I_{1}\right| & \leq \sum_{k=0}^{2 j-1} c_{k, 2 j}\left|f\left(t_{k}, y\left(t_{k}\right)\right)-f\left(t_{k}, y_{k}\right)\right| \leq \sum_{k=0}^{2 j-1} c_{k, 2 j} L\left|y\left(t_{k}\right)-y_{k}\right| \\
& \leq \frac{1}{\alpha} T^{\alpha} L \max _{0 \leq k \leq 2 j-1}\left|y\left(t_{k}\right)-y_{k}\right| .
\end{aligned}
$$

For $I I I_{1}$, we have, by Lemma 3.6 and the Lipschitz condition,

$$
\left|I I I_{1}\right| \leq c_{2 j, 2 j}\left|f\left(t_{2 j}, y\left(t_{2 j}\right)\right)-f\left(t_{2 j}, y_{2 j}^{P}\right)\right| \leq D_{3}^{\alpha} h^{\alpha} L\left|y\left(t_{2 j}\right)-y_{2 j}^{P}\right| .
$$

Now let us consider the bound for $\left|y\left(t_{2 j}\right)-y_{2 j}^{P}\right|$. We have 


$$
\begin{aligned}
& \Gamma(\alpha)\left(y\left(t_{2 j}\right)-y_{2 j}^{P}\right) \\
= & \int_{0}^{t_{2 j}}\left(t_{2 j}-u\right)^{\alpha-1} f(u, y(u)) d u-\left(\sum_{k=0}^{2 j-1} a_{k, 2 j} f\left(t_{k}, y_{k}\right)-a_{2 j, 2 j} f\left(t_{2 j}, t_{2 j}^{P P}\right)\right) \\
= & \left(\int_{0}^{t_{2 j}}\left(t_{2 j}-u\right)^{\alpha-1} f(u, y(u)) d u-\int_{0}^{t_{2 j}}\left(t_{2 j}-u\right)^{\alpha-1} P_{1}(u) d u\right) \\
& +\sum_{k=0}^{2 j-1} a_{k, 2 j}\left(f\left(t_{k}, y\left(t_{k}\right)\right)-f\left(t_{k}, y_{k}\right)\right)+a_{2 j, 2 j}\left(f\left(t_{2 j}, y\left(t_{2 j}\right)\right)-f\left(t_{2 j}, t_{2 j}^{P P}\right)\right) \\
= & I_{2}+I I_{2}+I I I_{2} .
\end{aligned}
$$

For $I_{2}$, we have, by Lemma 3.3,

$\left|I_{2}\right|=\left|\int_{0}^{t_{2 j}}\left(t_{2 j}-u\right)^{\alpha-1} f(u, y(u)) d u-\int_{0}^{t_{2 j}}\left(t_{2 j}-u\right)^{\alpha-1} P_{1}(u) d u\right| \leq C_{2}^{\alpha} T^{\alpha} h^{2}$.

For $I I_{2}$, we have, by Lemma 3.5 and the Lipschitz condition (3.1),

$$
\begin{aligned}
\left|I I_{2}\right| & \leq \sum_{k=0}^{2 j-1} a_{k, 2 j}\left|f\left(t_{k}, y\left(t_{k}\right)\right)-f\left(t_{k}, y_{k}\right)\right| \leq \sum_{k=0}^{2 j-1} a_{k, 2 j}\left|y\left(t_{k}\right)-y_{k}\right| \\
& \leq \frac{1}{\alpha} T^{\alpha} L_{0 \leq k \leq 2 j-1}\left|y\left(t_{k}\right)-y_{k}\right| .
\end{aligned}
$$

For $I I I_{2}$, we have, by Lemma 3.5 and Lipschitz condition (3.1),

$$
\left|I I I_{2}\right| \leq a_{2 j, 2 j}\left|f\left(t_{2 j}, y\left(t_{2 j}\right)\right)-f\left(t_{2 j}, y_{2 j}^{P P}\right)\right| \leq D_{2}^{\alpha} h^{\alpha} L\left|y\left(t_{2 j}\right)-y_{2 j}^{P P}\right| .
$$

We also need to consider the bound for $\left|y\left(t_{2 j}\right)-y_{2 j}^{P P}\right|$. We have

$$
\begin{aligned}
& \Gamma(\alpha)\left(y\left(t_{2 j}\right)-y_{2 j}^{P P}\right)=\int_{0}^{t_{2 j}}\left(t_{2 j}-u\right)^{\alpha-1} f(u, y(u)) d u-\sum_{k=0}^{2 j-1} b_{k, 2 j} f\left(t_{k}, y_{k}\right) \\
& =\int_{0}^{t_{2 j}}\left(t_{2 j}-u\right)^{\alpha-1} f(u, y(u)) d u-\sum_{k=0}^{2 j-1} b_{k, 2 j} f\left(t_{k}, y\left(t_{k}\right)\right) \\
& \quad+\sum_{k=0}^{2 j-1} b_{k, 2 j}\left(f\left(t_{k}, y\left(t_{k}\right)\right)-f\left(t_{k}, y_{k}\right)\right)=I_{3}+I I_{3} .
\end{aligned}
$$

For $I_{3}$, we have, by Lemma $3.2,\left|I_{3}\right| \leq C_{1}^{\alpha} T^{\alpha} h$.

For $I_{3}$, we have, by Lemma 3.5 and Lipschitz condition (3.1),

$$
\left|I_{3}\right| \leq \frac{1}{\alpha} T^{\alpha} L_{0 \leq k \leq 2 j-1}\left|y\left(t_{k}\right)-y_{k}\right| .
$$


Together these estimates, we have

$$
\begin{aligned}
& \Gamma(\alpha)\left|y\left(t_{2 j}\right)-y_{2 j}\right| \leq C_{3}^{\alpha} T^{\alpha} h^{3}+\frac{1}{\alpha} T^{\alpha} \max _{0 \leq k \leq 2 j-1}\left|y\left(t_{k}\right)-y_{k}\right| \\
& +D_{3}^{\alpha} h^{\alpha} L \frac{1}{\Gamma(\alpha)}\left(C_{2}^{\alpha} T^{\alpha} h^{2}+\frac{1}{\alpha} T^{\alpha} L \max _{0 \leq k \leq 2 j-1}\left|y\left(t_{k}\right)-y_{k}\right|\right. \\
& \left.+D_{2}^{\alpha} h^{\alpha} L \frac{1}{\Gamma(\alpha)}\left[C_{1}^{\alpha} T^{\alpha} h+\frac{1}{\alpha} T^{\alpha} L \max _{0 \leq k \leq 2 j-1}\left|y\left(t_{k}\right)-y_{k}\right|\right]\right) \\
& \leq\left[C_{3}^{\alpha} T^{\alpha} h^{3}+\frac{D_{3}^{\alpha} L C_{2}^{\alpha} T^{\alpha} h^{2+\alpha}}{\Gamma(\alpha)}+\frac{D_{3}^{\alpha} D_{2}^{\alpha} L^{2} C_{1}^{\alpha} T^{\alpha} h^{1+2 \alpha}}{\Gamma(\alpha)^{2}}\right] \\
& \quad+\left[\frac{1}{\alpha} T^{\alpha} L+\frac{D_{3}^{\alpha} L^{2}\left(\frac{1}{\alpha} T^{\alpha}\right) h^{\alpha}}{\Gamma(\alpha)}+\frac{D_{3}^{\alpha} D_{2}^{\alpha}\left(\frac{1}{\alpha} T^{\alpha}\right) L^{3} h^{2 \alpha}}{\Gamma(\alpha)^{2}}\right]_{0 \leq k \leq 2 j-1}\left|y\left(t_{k}\right)-y_{k}\right| .
\end{aligned}
$$

By mathematical induction (3.24), we have

$$
\begin{aligned}
\left|y\left(t_{2 j}\right)-y_{2 j}\right| \leq & {\left[\frac{C_{3}^{\alpha} T^{\alpha} h^{3}}{\Gamma(\alpha)}+\frac{D_{3}^{\alpha} L C_{2}^{\alpha} T^{\alpha} h^{2+\alpha}}{\Gamma(\alpha)^{2}}+\frac{D_{3}^{\alpha} D_{2}^{\alpha} L^{2} C_{1}^{\alpha} T^{\alpha} h^{1+2 \alpha}}{\Gamma(\alpha)^{3}}\right] } \\
& +\left[\frac{1}{\Gamma(\alpha+1)} T^{\alpha} L+\frac{D_{3}^{\alpha} L^{2}\left(\frac{1}{\alpha} T^{\alpha}\right) h^{\alpha}}{\Gamma(\alpha+1) \Gamma(\alpha)}+\frac{D_{3}^{\alpha} D_{2}^{\alpha}\left(\frac{1}{\alpha} T^{\alpha}\right) L^{3} h^{2 \alpha}}{\Gamma(\alpha+1) \Gamma(\alpha)^{2}}\right] C_{0} h^{3} .
\end{aligned}
$$

We first choose T sufficiently small, see Lemma 3.1 in [11] such that $\frac{1}{\Gamma(\alpha+1)} T^{\alpha} L \leq$ $\frac{1}{2}$. Then we fix this value for $\mathrm{T}$ and make the sum of the remaining terms in the right hand side of (3.25) smaller than $\frac{C_{0}}{2} h^{3}$ (for sufficiently small $h$ ) by choosing $C_{0}$ sufficiently large. Hence we obtain, for $1<\alpha \leq 2$,

$$
\left|y\left(t_{2 j}\right)-y_{2 j}\right| \leq \frac{C_{0}}{2} h^{3}+\frac{C_{0}}{2} h^{3}=C_{0} h^{3} .
$$

We also need to show that if (3.24) is true for $k=0,1,2, \ldots, 2 j$ with $j=1,2, \ldots, m-$ 1 , then it also holds for $k=2 j+1$. In fact, we have, with $j=1,2, \ldots, m-1$,

$$
\begin{aligned}
& \Gamma(\alpha)\left(y\left(t_{2 j+1}\right)-y_{2 j+1}\right)=\int_{0}^{t_{2 j+1}}\left(t_{2 j+1}-u\right)^{\alpha-1} f(u, y(u)) d u \\
& \quad-\left(\int_{0}^{t_{1}}\left(t_{2 j+1}-u\right)^{\alpha-1} f(u, y(u)) d u+\sum_{k=0}^{2 j-1} c_{k, 2 j} f\left(t_{k+1}, y_{k+1}\right)+c_{2 j, 2 j} f\left(t_{2 j+1}, y_{2 j+1}^{P}\right)\right) \\
& =\int_{t_{1}}^{t_{2 j+1}}\left(t_{2 j+1}-u\right)^{\alpha-1} f(u, y(u)) d u-\left(\sum_{k=0}^{2 j-1} c_{k, 2 j} f\left(t_{k+1}, y_{k+1}\right)+c_{2 j, 2 j} f\left(t_{2 j+1}, y_{2 j+1}^{P}\right)\right) \\
& =\left(\int_{t_{1}}^{t_{2 j+1}}\left(t_{2 j+1}-u\right)^{\alpha-1} f(u, y(u)) d u-\int_{t_{1}}^{t_{2 j+1}}\left(t_{2 j+1}-u\right)^{\alpha-1} Q_{2}(u) d u\right) \\
& +\sum_{k=0}^{2 j-1} c_{k, 2 j}\left(f\left(t_{k+1}, y\left(t_{k+1}\right)\right)-f\left(t_{k+1}, y_{k+1}\right)\right)+c_{2 j, 2 j}\left(f\left(t_{2 j+1}, y\left(t_{2 j+1}\right)\right)-f\left(t_{2 j+1}, y_{2 j+1}^{P}\right)\right)
\end{aligned}
$$

Using the same arguments as proving (3.26), we can show

$$
\left|y\left(t_{2 j+1}\right)-y_{2 j+1}\right| \leq C_{0} h^{3}, \quad j=1,2, \ldots, m-1 .
$$


Hence we complete the proof for the case where $1<\alpha \leq 2$.

Next we consider the case where $0<\alpha \leq 1$. Note that, by the assumptions, $\left|y\left(t_{0}\right)-y_{0}\right|=0,\left|y\left(t_{1}\right)-y_{1}\right|=0$. Assume that

$$
\left|y\left(t_{k}\right)-y_{k}\right| \leq C_{0} h^{1+2 \alpha}
$$

for $k=0,1,2, \ldots, 2 j-1, j=1,2, \ldots, m$. We must prove that this also holds for $k=$ $2 j$. In fact, by using the same arguments as showing (3.26), we get

$$
\begin{aligned}
\left|y\left(t_{2 j}\right)-y_{2 j}\right| \leq & {\left[\frac{{ }_{3}^{\alpha} T^{\alpha} h^{3}}{\Gamma(\alpha)}+\frac{D_{3}^{\alpha} L C_{2}^{\alpha} T^{\alpha} h^{2+\alpha}}{\Gamma(\alpha)^{2}}+\frac{D_{3}^{\alpha} D_{2}^{\alpha} L^{2} C_{1}^{\alpha} T^{\alpha} h^{1+2 \alpha}}{\Gamma(\alpha)^{3}}\right] } \\
& +\left[\frac{1}{\Gamma(\alpha+1)} T^{\alpha} L+\frac{D_{3}^{\alpha} L^{2}\left(\frac{1}{\alpha} T^{\alpha}\right) h^{\alpha}}{\Gamma(\alpha+1) \Gamma(\alpha)}+\frac{D_{3}^{\alpha} D_{2}^{\alpha}\left(\frac{1}{\alpha} T^{\alpha}\right) L^{3} h^{2 \alpha}}{\Gamma(\alpha+1) \Gamma(\alpha)^{2}}\right] C_{0} h^{1+2 \alpha} .
\end{aligned}
$$

As in the case for $1<\alpha \leq 2$, we first choose $\mathrm{T}$ sufficiently small such that $\frac{1}{\Gamma(\alpha+1)} T^{\alpha} L \leq \frac{1}{2}$. Then we fix this value for $\mathrm{T}$ and make the sum of the remaining terms in the right had side of (3.28) smaller than $\frac{C_{0}}{2} h^{1+2 \alpha}$ (for sufficiently small $h$ ) by choosing $C_{0}$ sufficiently large.

Hence we obtain, for $0<\alpha \leq 1$,

$$
\left|y\left(t_{2 j}\right)-y_{2 j}\right| \leq \frac{C_{0}}{2} h^{1+2 \alpha}+\frac{C_{0}}{2} h^{1+2 \alpha}=C_{0} h^{1+2 \alpha} .
$$

Similarly we can show that if (3.27) is true for $k=0,1,2, \ldots, 2 j$ with $j=1,2, \ldots, m-$ 1 , then it is also true for $k=2 j+1$. Together these estimates complete the proof of Theorem 3.1.

Remark 3.2 In Theorem 3.1, we require that ${ }_{0}^{C} D_{t}^{\alpha} y(t)$ is in $C^{3}[0, T]$ which implies that the solution has the form, see Page 46 in [11],

$$
y(t)=c t^{\alpha}+\text { smoother terms. }
$$

Since $y(t)$ has low regularity at $t=0$, it may be necessary to use some high order methods and a small step size to approximate the solutions near $t=0$ and to use a big step size to calculate the approximate solutions at other nodes to get the required accuracy. We will investigate this interesting issue in our future work.

\section{Numerical simulations}

Example 4.1 [7] Consider

$$
\begin{aligned}
& { }_{0}^{C} D_{t}^{\alpha} y(t)=\beta y(t)+f(t), \quad t \in[0,1], \\
& y(0)=y_{0},
\end{aligned}
$$

where $y_{0}=0,0<\alpha<1, \beta=-1$ and $f(t)=\left(t^{2}+2 t^{2-\alpha} / \Gamma(3-\alpha)\right)+\left(t^{3}+3 ! t^{3-\alpha} / \Gamma(4-\right.$ $\alpha)$ ). The exact solution is $y(t)=t^{2}+t^{3}$. 
The main purpose is to check the order of convergence of the numerical method with respect to the fractional order $\alpha$. For various choices of $\alpha \in(0,1)$, we computed the errors at $t=1$. We choose the step size $h=1 /\left(5 \times 2^{l}\right), l=1,2, \ldots, 7$, i.e, we divided the interval $[0,1]$ into $n=1 / h$ small intervals with nodes $0=t_{0}<t_{1}<\cdots<$ $t_{n}=1$. Then we compute the error $e\left(t_{n}\right)=y\left(t_{n}\right)-y_{n}$. By Theorem 2.22, we have

$$
\left|e\left(t_{n}\right)\right|=\left|y\left(t_{n}\right)-y_{n}\right| \leq C h^{3-\alpha},
$$

To observe the order of convergence we shall compute the error $\left|e\left(t_{n}\right)\right|$ at $t_{n}=1$ for the different values of $h$. Denote $\left|e_{h}\left(t_{n}\right)\right|$ the error at $t_{n}=1$ for the step size $h$. Let $h_{l}=h=1 /\left(5 \times 2^{l}\right)$ for a fixed $l=1,2, \ldots, 7$. We then have

$$
\frac{\left|e_{h_{l}}\left(t_{n}\right)\right|}{\left|e_{h_{l+1}}\left(t_{n}\right)\right|} \approx \frac{C h_{l}^{3-\alpha}}{C h_{l+1}^{3-\alpha}}=2^{3-\alpha}
$$

which implies that the order of convergence satisfies $3-\alpha \approx \log _{2}\left(\frac{\left|e_{h_{l}}\left(t_{n}\right)\right|}{\left|e_{h_{l+1}}\left(t_{n}\right)\right|}\right)$. In Table 4.1, we compute the orders of convergnce for the different values of $\alpha$. The numerical results are consistent with the theoretical results.

\begin{tabular}{|c|c|c|c|}
\hline$n$ & ERC $(\alpha=0.25)$ & ERC $(\alpha=0.5)$ & ERC $(\alpha=0.75)$ \\
\hline 10 & & & \\
\hline 20 & 2.7486 & 2.4840 & 2.2070 \\
\hline 40 & 2.7526 & 2.4937 & 2.2304 \\
\hline 80 & 2.7544 & 2.4979 & 2.2408 \\
\hline 160 & 2.7549 & 2.4997 & 2.2456 \\
\hline 320 & 2.7547 & 2.5003 & 2.2479 \\
\hline 640 & 2.7543 & 2.5005 & 2.2490 \\
\hline
\end{tabular}

Table 4.1 Numerical results at $t=1$ for $\beta=-1$

$$
\text { and } f(t)=\left(t^{2}+2 t^{(2-\alpha)} / \Gamma(3-\alpha)\right)+\left(t^{3}+3 ! t^{3-\alpha} / \Gamma(4-\alpha)\right)
$$

In Figure 4.1, we plot the order of the convergence. We have from (4.3)

$$
\log _{2}\left(\left|e\left(t_{n}\right)\right|\right) \leq \log _{2}(C)+(3-\alpha) \log _{2}(h) .
$$

Let $y=\log _{2}\left(\left|e\left(t_{n}\right)\right|\right)$ and $x=\log _{2}(h)$. In Figure 4.1, we plot the function $y=y(x)$ for the different values of $x=\log _{2}(h)$ where $h=1 /\left(5 \times 2^{l}\right), l=1,2, \ldots, 7$. To observe the order of convergence, we also plot the straight line $y=(3-\alpha) x$, where $\alpha=0.75$. We see that these two lines are exactly parallel which means that the order of convergence of the numerical method is $O\left(h^{3-\alpha}\right)$.

Example 4.2 [11] Our second example deals with the nonlinear fractional differential equation where the unknown solution $y$ has a smooth derivative of order $\alpha$. Specifically we shall look at the equation [11]

${ }_{0}^{C} D_{t}^{\alpha} y(t)=\frac{40320}{\Gamma(9-\alpha)} t^{8-\alpha}-3 \frac{\Gamma(5+\alpha / 2)}{\Gamma(5-\alpha / 2)} t^{4-\alpha / 2}+\frac{9}{4} \Gamma(\alpha+1)+\left(\frac{3}{2} t^{\alpha / 2}-t^{4}\right)^{3}-[y(t)]^{3 / 2}$. 


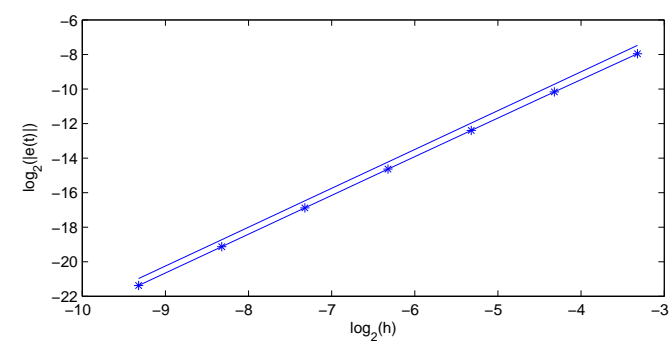

Fig. 4.1 The experimentally determined orders of convergence ("EOC") at $t=1$ in Example 4.2 with $\alpha=0.75$

The initial conditions were chosen to be homogeneous $\left(y(0)=0, y^{\prime}(0)=0\right.$; the latter only in the case $1<\alpha<2$ ). This equation has been chosen because it exhibits a difficult (nonlinear and nonsmooth) right-hand side, and yet we are able to find its exact solution, thus allowing us to compare the numerical results for this nontrivial case to the exact results. Indeed, the exact solution of this initial value problem is

$$
y(t)=t^{8}-3 t^{4+\alpha / 2}+\frac{9}{4} t^{\alpha}
$$

and hence

$$
{ }_{0}^{C} D_{t}^{\alpha} y(t)=\frac{40320}{\Gamma(9-\alpha)} t^{8-\alpha}-3 \frac{\Gamma(5+\alpha / 2)}{\Gamma(5-\alpha / 2)} t^{4-\alpha / 2}+\frac{9}{4} \Gamma(\alpha+1),
$$

which implies ${ }_{0}^{C} D_{t}^{\alpha} y \in C^{3}[0, T]$ for arbitrary $T>0$ and $0<\alpha \leq 2$, and thus the conditions of Theorem 3.1 are fulfilled.

For various choices of $\alpha \in(0,2]$, we compute the errors at $t_{n}=1$. We choose the step size $h=1 /\left(5 \times 2^{l}\right), l=1,2, \ldots, 7$, i.e, we divided the interval $[0,1]$ into $n=1 / h$ small intervals with nodes $0=t_{0}<t_{1}<\cdots<t_{n}=1$. Then we compute the error $e\left(t_{n}\right)=y\left(t_{n}\right)-y_{n}$. By Theorem 3.1, we have

$$
\max _{0 \leq k \leq 2 m}\left|y\left(t_{k}\right)-y_{k}\right| \leq\left\{\begin{array}{l}
C_{0} h^{1+2 \alpha}, \quad \text { if } 0<\alpha \leq 1, \\
C_{0} h^{3}, \quad \text { if } 1<\alpha \leq 2 .
\end{array}\right.
$$

In Table 4.2, we compute the orders of convergence for different values of $\alpha$. We observe that the order of convergence is $O\left(h^{1+2 \alpha}\right)$ for $0<\alpha \leq 1$. But the observed order of convergence is higher than 3 for $1<\alpha \leq 2$ in this example. For example, when $\alpha=1.25$, the experimentally determined order is 3.5 . When $\alpha=1.75$, the experimentally determined order is almost 4 .

In Figure 4.2, we plot the order of convergence. We have

$$
\log _{2}\left(\left|e\left(t_{n}\right)\right|\right) \leq \log _{2}(C)+(1+2 \alpha) \log _{2}(h) .
$$

Let $y=\log _{2}\left(\left|e\left(t_{n}\right)\right|\right)$ and $x=\log _{2}(h)$. In Figure 4.2, we plot the function $y=y(x)$ for the different values of $x=\log _{2}(h)$ where $h=1 /\left(5 \times 2^{l}\right), l=1,2, \ldots, 7$. To observe the order of convergence, we also plot the straight line $y=(1+2 \alpha) x$, where $\alpha=$ 


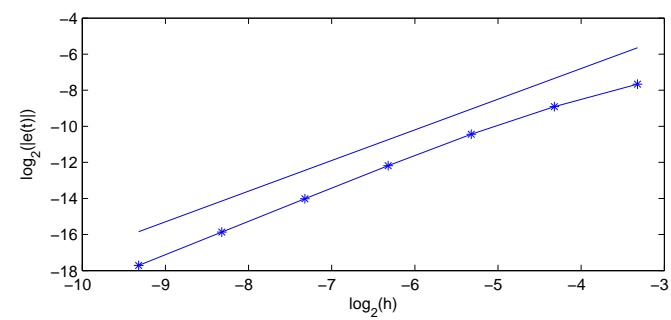

Fig. 4.2 The experimentally determined orders of convergence ("EOC") at $t=1$ in Example 4.2 with $\alpha=0.35$

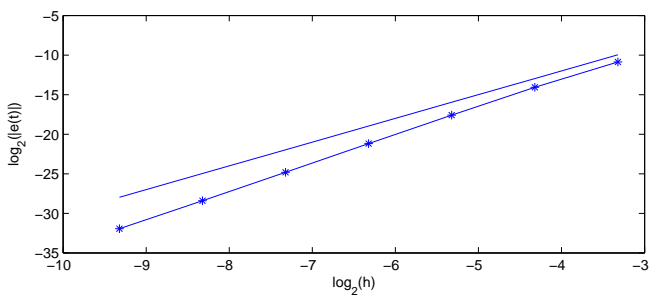

Fig. 4.3 The experimentally determined orders of convergence ("EOC ") at $t=1$ in Example 4.2 with $\alpha=1.25$

0.35. We see that these two lines are almost parallel which confirms that the order of convergence of the numerical method is $O\left(h^{1+2 \alpha}\right)$.

In Figure 4.3, we will plot the order of convergence for $\alpha=1.25$. We plot the function $y=y(x)$ for the different values of $x=\log _{2}(h)$ where $h=1 /\left(5 \times 2^{l}\right), l=$ $1,2, \ldots, 7$. To observe the order of convergence, we also plot the straight line $y=3 x$. We observe that the order of convergence is higher than 3 ( almost $1+2 \alpha)$.

\begin{tabular}{|c|c|c|c|}
\hline$n$ & ERC $(\alpha=0.35)$ & ERC $(\alpha=1.25)$ & ERC $(\alpha=1.75)$ \\
\hline 10 & & & \\
\hline 20 & 1.2475 & 3.2109 & 3.8503 \\
\hline 40 & 1.5302 & 3.5125 & 3.9305 \\
\hline 80 & 1.7461 & 3.6050 & 3.9633 \\
\hline 160 & 1.8293 & 3.6182 & 3.9786 \\
\hline 320 & 1.8518 & 3.5886 & 3.9856 \\
\hline 640 & 1.8478 & 3.5422 & 4.0225 \\
\hline
\end{tabular}

Table 4.2 Numerical results at $t=1$ in Example 4.2 with the different fractional order $\alpha$

Acknowledgements The authors wish to thank Kai Diethelm for reading the first version of this paper and making useful suggestions. The authors also wish to thank the anonymous reviewers of this paper for their careful reading and useful comments. 


\section{References}

1. L. Blank, Numerical treatment of differential equations of fractional order, Nonlinear world, 4(1997), 473-491.

2. J. Cao and C. Xu, A high order schema for the numerical solution of the fractional ordinary differential equations, J. Comp. Phys., 238(2013), 154-168.

3. W. H. Deng, Numerical algorithm for the time fractional Fokker-Planck equation, J. Comp. Phys., 227(2007), 1510-1522.

4. W. H. Deng, Short memory principle and a predict-corrector approach for fractional differential equations, J. Comput. Appl. Math., 206(2007), 1768-1777.

5. W. H. Deng and C. Li, Numerical schemes for fractional ordinary differential equations, Numerical Modelling, edited by: Prof. Peep Miidla, Chapter 16, 355-374, Publisher InTech, 2012.

6. K. Diethelm, Generalized compound quadrature formulae for finite-part integrals, IMA J. Numer. Anal., 17(1997), 479-493.

7. K. Diethelm, An algorithm for the numerical solution of differential equations of fractional order, Electronic Transactions on Numerical Analysis, 5(1997), 1-6.

8. K. Diethelm, The Analysis of Fractional Differential Equations, An Application-Oriented Using Differential Operators of Caputo Type, Lecture Notes in Mathematics, Vol. 2004, Springer, 2010.

9. K. Diethelm, Monotonicity results for a compound quadrature method for finite-part integrals, Journal of Inequalities in Pure and Applied Mathematics, 5 (2004), issue 2, article 44.

10. K. Diethelm and N. J. Ford, Analysis of fractional differential equations, Journal of Mathematics Analysis and Application, 265(2002), 229-248.

11. K. Diethelm, N. J. Ford, and A.D. Freed, Detailed error analysis for a fractional Adams method, Numerical Algorithms, 36(2004), 31-52.

12. K. Diethelm, N. J. Ford, and A. D. Freed, A predictor-corrector approach for the numerical solution of fractional differential equations, Nonlinear Dynam., 29(2002), 3-22.

13. K. Diethelm and Y. Luchko, Numerical solution of linear multi-term initial value problems of fractional order, J. Comput. Anal. Appl., 6(2004), 243-263.

14. K. Diethelm and G. Walz, Numerical solution of fractional order differential equations by extrapolation, Numer. Algorithms, 16(1997), 231-253.

15. N. J. Ford and A. C. Simpson, The numerical solution of fractional differential equations: speed versus accuracy, Numer. Algorithms, 26(2001), 333-346.

16. N. J. Ford, M. L. Morgado, M. Rebelo, Nonpolynomial collocation approximation of solutions to fractional differential equations, Fract. Calc. Appl. Anal., to appear, 2013.

17. R. Gorenflo, Fractional Calculus: Some Numerical Methods, CISM Lecture Notes, 1996.

18. C. Lubich, Convolution quadrature and discretized operational calculus II, Num. Math., 52(1988), 413-425.

19. Y. Luchko and R. Gorenflo, The initial value problem for some fractional differential equations with the Caputo derivatives, Preprint Serie A 08-98, Fachbereich Mathematik und Informatik, Freie Universität Berlin, Berlin, 1998.

20. J. N. Lyness, Finite-part integration and the Euler-MacLaurin expansion. In R. V. M. Zahar (ed.): Approximation and Computation, Internat. Ser. Numer. Math. 119, Birkhäuser, Basel, 397-407, 1994.

21. I. Podlubny, Fractional Differential Equations, Academic Press, San Diego, 1999.

22. L. Zhao and W. H. Deng, Jacobi-predictor-corrector approach for the fractional ordinary differential equations, arXiv:1201.5952v2, 2012. 